\title{
FLAT PARABOLIC VECTOR BUNDLES ON ELLIPTIC CURVES
}

\author{
THIAGO FASSARELLA AND FRANK LORAY
}

\begin{abstract}
We describe the moduli space of logarithmic rank 2 connections on elliptic curves with 2 poles.
\end{abstract}

\section{Contents}

1. Introduction

2. Existence of logarithmic connections

2.1. Logarithmic connections

2.2. Flatness criterion over elliptic curves

2.3. Indecomposable quasi-parabolic bundles

3. Moduli space of connections on elliptic curves with two poles

3.1. Moduli space of parabolic vector bundles

3.2. Fiber compactification of the moduli space and Higgs fields 14

3.3. From logarithmic connections to fuchsian systems with five poles 15

3.4. Universal family of fuchsian systems 18

3.5. An open set of the moduli space 21

3.6. Whole moduli space of connections 24

3.7. Apparent singularities 29

3.8. Symplectic structure and Torelli phenomenon 32

4. Appendix 34

4.1. Parabolic vector bundles 34

4.2. Elementary transformations 35

4.3. Endomorphisms of quasi-parabolic vector bundles 36

4.4. The case of elliptic curves 36

4.5. Moduli space of connections 38

References 40

\section{INTRODUCTION}

In this paper, we investigate the geometry of certain moduli spaces of connections on curves $C$. We consider pairs $(E, \nabla)$ where $E \rightarrow C$ is a rank 2 vector bundle and $\nabla: E \rightarrow E \otimes \Omega_{C}^{1}(D)$ is a logarithmic connection with (reduced) polar divisor $D=t_{1}+\cdots+t_{n}$. Once we have prescribed the base curve $(C, D)$, the trace connection $(\operatorname{det}(E), \operatorname{tr}(\nabla))$ and the eigenvalues $\nu=\left(\nu_{1}^{ \pm}, \ldots, \nu_{n}^{ \pm}\right)$of the residual

2010 Mathematics Subject Classification. Primary 34M55; Secondary 14D20, 32G2032G34. Key words and phrases: logarithmic connection, parabolic structure, elliptic curve, apparent singularities, symplectic structure. The first author is supported by CNPq, Proc. 234895/2013-6. The second author is supported by CNRS, ANR-16-CE40-0008 Foliage. The authors also thank Brazilian-French Network in Mathematics and MATH AmSud for support. 
connection matrix at each pole, then we can define the moduli space $\operatorname{Con}^{\nu}(C, D)$ of those pairs $(E, \nabla)$ up to isomorphism. For a generic choice of $\nu$ (compatible with $\operatorname{tr}(\nabla))$ all connections $(E, \nabla)$ are irreducible and the moduli $\operatorname{Con}^{\nu}(C, D)$ can be constructed as a GIT quotient (see [17]): it is a smooth irreducible quasi-projective variety of dimension $2 N$ where $N=3 g-3+n$ is the dimension of deformation of the base curve and $g$ denotes the genus of the curve. Moreover, the variety $\operatorname{Con}^{\nu}(C, D)$ admits a holomorphic symplectic structure (see [5]) which turns to be algebraic (see [10, 8) : there is a rational 2-form $\omega$ which is regular and having maximal rank $N$ on $\operatorname{Con}^{\nu}(C, D)$.

It is natural to consider the forgetful map $\pi:(E, \nabla) \mapsto(E, \mathbf{p})$ which to a connection associates the underlying parabolic bundle: the parabolic data $\mathbf{p}=$ $\left(p_{1}, \ldots, p_{n}\right)$ consists of the $\nu_{i}^{+}$-eigenline $\left.p_{i} \subset E\right|_{t_{i}}$ for each pole. The moduli space $\operatorname{Bun}(C, D)$ of those parabolic bundles admitting a connection is $N$-dimensional and the map $\pi$ above turns to be Lagrangian, i.e. its fibers are Lagrangian submanifolds. However, $\operatorname{Bun}(C, D)$ is not a variety, but a non Hausdorff scheme; it is a finite union of projective varieties patched together along Zariski open sets. Over the open subset of simple bundles (i.e. without automorphisms), the Lagrangian fibration $\pi$ is an affine $\mathbb{A}^{N}$-bundle whose linear part is the cotangent bundle $T^{*} \operatorname{Bun}(C, D)$, and the symplectic structure comes from Liouville form. It is mainly this heuristic picture that we want to describe in a particular case.

The picture is very well known in the case $(g, n)=(0,4)$, since $\operatorname{Con}^{\nu}(C, D)$ corresponds to the Okamoto space of initial conditions for Painlevé VI equation in this case (see [9]). The case $(0, n)$ has been studied in [1, 18, 13, 11] and corresponds to Garnier systems. The case $(1,1)$ has been studied in [12, where it is shown to be equivalent to the Painlevé $(0,4)$ case with particular exponents, due to hyperellipticity of the curve. Similarly, the case $(2,0)$ is studied in [7] and turns to be the quotient of the Garnier case $(0,6)$ by an involution, again by hyperellipticity. The case $(1,2)$ involved in the present paper is the first one that does not reduce to genus zero case: for generic eigenvalues $\nu$, the hyperelliptic involution does not preserve the spectral data.

Results. We fix $C$ to be the elliptic curve with affine equation $y^{2}=x(x-$ 1) $(x-\lambda), \lambda \in \mathbb{C} \backslash\{0,1\}$, and denote by $w_{\infty} \in C$ the point at infinity. In Section 2 the number $n=\operatorname{deg}(D)$ of poles and eigenvalues $\nu$ are arbitrary. There, we study which parabolic bundles $(E, \mathbf{p})$ over $(C, D)$ are $\nu$-flat, i.e. admit a connection $\nabla$ with prescribed trace and exponents, compatible with parabolics. This has been done for a general curve in [4] in the orbifold case (i.e. with rational eigenvalues) and we extend his criterion for general eigenvalues in Theorem 2.2.

Theorem 1. A parabolic bundle $(E, \boldsymbol{p})$ over an elliptic curve $(C, D)$ is $\nu$-flat if, and only if, it satisfies

$$
\text { Fuchs relation: } \operatorname{deg}(E)+\sum_{k=1}^{n}\left(\nu_{k}^{+}+\nu_{k}^{-}\right)=0
$$

and for each decomposition $(E, \boldsymbol{p})=\left(L^{\prime}, \boldsymbol{p}^{\prime}\right) \oplus\left(L^{\prime \prime}, \boldsymbol{p}^{\prime \prime}\right)$, we have

$$
\operatorname{deg}(L)+\sum_{p_{k} \in L} \nu_{k}^{+}+\sum_{p_{k} \notin L} \nu_{k}^{-}=0
$$

for $L=L^{\prime}$ and $L^{\prime \prime}$. 
For generic $\nu$ satisfying Fuchs relation (and $n>0$ ), the second identity cannot occur and in that case we have:

$$
\nu \text {-flat } \Leftrightarrow \text { indecomposable. }
$$

For $n=2$, the moduli space of indecomposable bundles has been recently described in [6. It is a non Hausdorff scheme $X$ whose Hausdorff quotient is $\mathbb{P}^{1} \times \mathbb{P}^{1}$. More precisely, there is an embedding $C \stackrel{\sim}{\rightarrow} \Gamma \subset \mathbb{P}^{1} \times \mathbb{P}^{1}$ as a bidegree $(2,2)$ curve, such that $X$ is the union of two copies $X_{<}$and $X_{>}$of $\mathbb{P}^{1} \times \mathbb{P}^{1}$ patched together outside the curve $\Gamma$. For instance, if we fix $\operatorname{det}(E)=\mathcal{O}_{C}\left(w_{\infty}\right)$, then $X_{<}$will correspond to those parabolic bundles $(E, \mathbf{p})$ such that $E=E_{1}$ is the unique non trivial extension

$$
0 \longrightarrow \mathcal{O}_{C} \longrightarrow E_{1} \longrightarrow \mathcal{O}_{C}(w) \longrightarrow 0
$$

Then, the missing $\nu$-flat bundles occurring in $X_{>}$are those decomposable $E$ with indecomposable parabolic structure. More details are recalled in Section 3.1 .

We denote by $\operatorname{Con}_{<}^{\nu}(C, D)$ the open subset of $\operatorname{Con}^{\nu}(C, D)$ over $X_{<}$. In Section 3.4, an explicit universal family of connections is given for $\operatorname{Con}_{<}^{\nu}(C, D)$ : through a birational trivialization $E_{1} \rightarrow \mathcal{O}_{C} \times \mathcal{O}_{C}$, it is given by an explicit family of Fuchsian system with 3 additional apparent singular points. To set our main result, given a parabolic bundle $(E, \mathbf{p})$, let us introduce the parabolic structure $\mathbf{p}^{-}=\left(p_{1}^{-}, p_{2}^{-}\right)$ associated to $\nu_{k}^{-}$-eigenspaces, and denote $\mathbf{p}^{+}:=\mathbf{p}$. Then, we have a natural map

$$
\text { Par : }\left\{\begin{array}{ccc}
\operatorname{Con}_{<}^{\nu}(C, D) & \rightarrow & X_{<} \times X_{<} \\
\left(E_{1}, \nabla\right) & \mapsto & \left(\left(E_{1}, \mathbf{p}^{+}\right),\left(E_{1}, \mathbf{p}^{-}\right)\right)
\end{array}\right.
$$

Since $\nu$ is generic, we can assume $\nu_{k}^{+} \neq \nu_{k}^{-}$for $k=1,2$, and therefore $p_{k}^{+} \neq p_{k}^{-}$. This implies that the image of Par in $X_{<} \times X_{<}$avoid the "incidence variety"

$$
I:=\left\{z_{1}=\zeta_{1}\right\} \cup\left\{z_{2}=\zeta_{2}\right\} \subset \underbrace{\left(\mathbb{P}_{z_{1}}^{1} \times \mathbb{P}_{z_{2}}^{1}\right) \times\left(\mathbb{P}_{\zeta_{1}}^{1} \times \mathbb{P}_{\zeta_{2}}^{1}\right)}_{X<\times X_{<}} .
$$

Setting $\nu_{k}:=\nu_{k}^{+}-\nu_{k}^{-}$, our main result is (see Theorem 3.8 and Section 3.8)

Theorem 2. If $\nu_{1} \cdot \nu_{2} \neq 0$, then the map

$$
\text { Par: } \operatorname{Con}_{<}^{\nu}(C, D) \rightarrow X_{<} \times X_{<}
$$

induces an isomorphism onto the complement of the incidence variety $X_{<} \times X_{<} \backslash I$ and the image of the symplectic structure is given by

$$
\omega=-\frac{1}{2}\left\{\nu_{1} \frac{d z_{1} \wedge d \zeta_{1}}{\left(z_{1}-\zeta_{1}\right)^{2}}+\nu_{2} \frac{d z_{2} \wedge d \zeta_{2}}{\left(z_{2}-\zeta_{2}\right)^{2}}\right\} .
$$

In the spirit of classical Torelli Theorem, this shows that exponents (difference of eigenvalues) can be read off from the moduli space, see Proposition 3.20 This completes the result of $[\underline{6}$. Theorem B] where it is shown that the moduli space of $\nu$-flat bundles keeps track of the punctured curve $(C, D)$.

We investigate in Section 3.6 .3 how to cover the full moduli space $\operatorname{Con}^{\nu}(C, D)$ by three charts like the one in Theorem 2, see Theorem 3.14. Finally, in Section 3.7 (see also Section [3.8), we study the "apparent map" which, to a connection $(E, \nabla) \in \operatorname{Con}_{<}^{\nu}(C, D)$, associates the position of the apparent singular points of the corresponding scalar equation via the "cyclic vector" $\mathcal{O}_{C} \subset E_{1}$ (see [10, 13] for instance). This gives us a map

$$
A p p: \operatorname{Con}_{<}^{\nu}(C, D) \rightarrow\left|\mathcal{O}_{C}\left(w_{\infty}+t_{1}+t_{2}\right)\right| \simeq \mathbb{P}^{2}
$$


which turns out to be Lagrangian. Similarly as [13, Theorem 1.1] in genus zero case, we have

Theorem 3. If $\nu_{1}+\nu_{2}+1 \neq 0$, then the rational map

$$
\text { Bun } \times A p p: \operatorname{Con}_{<}^{\nu}(C, D) \longrightarrow\left(\mathbb{P}_{z_{1}}^{1} \times \mathbb{P}_{z_{2}}^{1}\right) \times \mathbb{P}^{2}
$$

is birational. More precisely, the restriction

$$
\text { App : } \operatorname{Bun}^{-1}(\boldsymbol{p}) \longrightarrow \mathbb{P}^{2}
$$

is injective if, and only if, $\boldsymbol{p} \in X_{<} \simeq \mathbb{P}_{z_{1}}^{1} \times \mathbb{P}_{z_{2}}^{1}$ is lying outside $\left\{z_{1}=t\right\} \cup\left\{z_{2}=t\right\}$, where $t$ is the first coordinate of $t_{1}$.

Notation and conventions. Curves are always assumed to be irreducible and defined over the field $\mathbb{C}$ of complex numbers. Given a projective smooth curve $C$ and a holomorphic vector bundle $E \longrightarrow C$ over $C$ we make no difference in notation between the total space $E$ and its locally free sheaf defined by holomorphic sections. We denote by $E^{*}$ the sheaf $\mathcal{H o m}_{\mathcal{O}_{C}}\left(E, \mathcal{O}_{C}\right)$. If $E$ and $F$ are two vector bundles over $C$ we denote by $\operatorname{Hom}(E, F)$ the space of global sections of the sheaf $\mathcal{H} m_{\mathcal{O}_{X}}(E, F)$. In particular, $\operatorname{End}(E)=\operatorname{Hom}(E, E)$ denotes the set of endomorphisms of $E$. We use the notation $\mathbb{P}_{z}^{1}$ to denote the projective line $\mathbb{P}^{1}$ equipped with an affine coordinate $z \in \mathbb{C}$; this notation will be used to distinguish between several occurences of $\mathbb{P}^{1}$.

\section{EXISTENCE OF LOGARITHMiC CONNECTIONS}

In this section, we shall investigate the existence of logarithmic connections on a given rank 2 quasi-parabolic vector bundle $(E, \mathbf{p})$ over an elliptic curve $C$. We give a criterion in Theorem 2.2 which extends the famous Weil criterion [21] for holomorphic connections, and Biswas criterion 4 for logarithmic connections with rational residual eigenvalues.

2.1. Logarithmic connections. Let us fix a set of $n$ distinct points $\mathbf{t}=\left\{t_{1}, \ldots, t_{n}\right\}$ on a smooth projective complex curve $C$ and let $D=t_{1}+\cdots+t_{n}$ be the reduced effective divisor associated to it. A logarithmic connection on a vector bundle $E$ over $C$ with polar divisor $D$ is a $\mathbb{C}$-linear map

$$
\nabla: E \longrightarrow E \otimes \Omega_{C}^{1}(D)
$$

satisfying the Leibniz rule

$$
\nabla(f \cdot s)=d f \otimes s+f \cdot \nabla(s)
$$

for any local section $s$ of $E$ and function $f$ on $C$. If $E$ is of rank $r$, then it is given by a cocycle $\left\{G_{i j}\right\} \in \mathrm{H}^{1}\left(C, G L\left(r, \mathcal{O}_{C}\right)\right)$ defined by an atlas of trivializations $C=\cup U_{i}$ where $\left.E\right|_{U_{i}} \simeq U_{i} \times \mathbb{C}^{r}$. Locally, over each $U_{i}$, the connection writes $\nabla=d_{C}+A_{i}$ where $d_{C}: \mathcal{O}_{C} \longrightarrow \Omega_{C}^{1}$ is the differential operator on $C$ and $A_{i}$ is a $r \times r$ matrix with coefficients in $\Omega_{C}^{1}(D)$ that glue together through the transition map. That is, $A_{i}$ is a $r \times r$ matrix of meromorphic 1-forms having at most simple poles on $\mathbf{t}$ and this collection of matrices must satisfies

$$
A_{j}=G_{i j} \cdot A_{i} \cdot G_{i j}^{-1}+d G_{i j} \cdot G_{i j}^{-1}
$$

over intersections $U_{i} \cap U_{j}$. For each pole $t_{k} \in U_{i}$, the residue homomorphism $\operatorname{Res}_{t_{k}}(\nabla)=\operatorname{Res}_{t_{k}}\left(A_{i}\right) \in \operatorname{End}\left(\left.E\right|_{t_{k}}\right)$ is well defined. If $E$ is of rank 2, then let $\nu_{k}^{+}$ 
and $\nu_{k}^{-}$be the eigenvalues of $\operatorname{Res}_{t_{k}}(\nabla)$, called local exponents of $\nabla$ over $t_{k}$. The data

$$
\nu=\left(\nu_{1}^{ \pm}, \ldots, \nu_{n}^{ \pm}\right) \in \mathbb{C}^{2 n}
$$

is called local exponent of $\nabla$. We note that the connection $\nabla$ induces a logarithmic connection $\operatorname{tr}(\nabla)$ on the determinant line bundle $\operatorname{det}(E)$ with

$$
\operatorname{Res}_{t_{k}}(\operatorname{tr}(\nabla))=\nu_{k}^{+}+\nu_{k}^{-} \text {. }
$$

By Residue Theorem we obtain the Fuchs relation

$$
d+\sum_{k=1}^{n}\left(\nu_{k}^{+}+\nu_{k}^{-}\right)=0 .
$$

where $d=\operatorname{deg}(E)$.

When $E$ admits a holomorphic connection, then it is called flat. There is a similar notion for quasi-parabolic vector bundles. We fix $\left(E, \mathbf{p}=\left\{p_{k}\right\}\right)$ a quasi-parabolic rank 2 vector bundle over $(C, \mathbf{t})$, see Section 4.1. We shall say that $(E, \mathbf{p})$ is $\nu$-flat if it admits a logarithmic connection $\nabla$ with given local exponent $\nu$ satisfying

$$
\operatorname{Res}_{t_{k}}(\nabla)\left(p_{k}\right)=\nu_{k}^{+} \cdot p_{k} .
$$

We also say that $(E, \nabla, \mathbf{p})$ is a $\nu$-parabolic connection.

The following flatness criterion for vector bundles over curves is due to A. Weil (see [21, 2]): a vector bundle is flat if, and only if, it is the direct sum of indecomposable vector bundles of degree zero. A generalization to the parabolic context of the Weil's criterion was obtained in 4 where the local exponents are supposed to be rational numbers. Besides that, when $C=\mathbb{P}^{1}$ and $\nu$ is generic

$$
\sum_{k=1}^{n} \nu_{k}^{\epsilon_{k}} \notin \mathbb{Z} ; \quad \epsilon_{k} \in\{+,-\}
$$

the following equivalent conditions are known (cf. [1]):

(1) $(E, \mathbf{p})$ is $\nu$-flat;

(2) $\operatorname{End}_{0}(E, \mathbf{p})=0$

(3) $(E, \mathbf{p})$ is indecomposable.

Remark 2.1. We note that if $C$ is an elliptic curve then the above conditions (1) and (2) are not equivalents. For instance, let $E_{0}$ be the unique non trivial extension

$$
0 \longrightarrow \mathcal{O}_{C} \longrightarrow E_{0} \longrightarrow \mathcal{O}_{C} \longrightarrow 0 .
$$

If $\mathbf{p}$ is a parabolic structure with all parabolics lying on $\mathcal{O}_{C} \hookrightarrow E_{0}$, then from Proposition 4.4

$$
\operatorname{End}_{0}\left(E_{0}, \mathbf{p}\right)=\mathbb{C} .
$$

But $\left(E_{0}, \mathbf{p}\right)$ is indecomposable and, as we will see in Theorem 2.2. it is $\nu$-flat for any $\nu$ satisfying the Fuchs Relation.

2.2. Flatness criterion over elliptic curves. In this section, we will obtain a parabolic version of Weil's criterion over elliptic curves, in the same spirit of 1, 4] (see Theorem 2.2).

Before that, let us recall the definition of direct summand as well as parabolic degree. Let $(E, \mathbf{p})$ be a rank 2 quasi-parabolic bundle. We say that $(L, \mathbf{q})$ is a 
direct summand of $(E, \mathbf{p})$ if either $(E, \mathbf{p})=(L, \mathbf{q})$ or $L$ is a line bundle and there exists another parabolic line bundle, say $(M, \mathbf{r})$, such that

$$
(E, \mathbf{p})=(L, \mathbf{q}) \oplus(M, \mathbf{r}) .
$$

Its parabolic degree, denoted by $\operatorname{deg}_{\nu}(L, \mathbf{q})$, with respect to $\nu=\left(\nu_{1}^{ \pm}, \ldots, \nu_{n}^{ \pm}\right)$is defined as follows:

- if $(E, \mathbf{p})=(L, \mathbf{q})$ then we set

$$
\operatorname{deg}_{\nu}(L, \mathbf{q}):=\operatorname{deg}(E)+\sum_{k=1}^{n}\left(\nu_{k}^{+}+\nu_{k}^{-}\right) ;
$$

- and if $(E, \mathbf{p})=(L, \mathbf{q}) \oplus(M, \mathbf{r})$ then it is defined by

$$
\operatorname{deg}_{\nu}(L, \mathbf{q}):=\operatorname{deg}(L)+\sum_{k=1}^{n} \nu_{k}^{\epsilon_{k}}
$$

where $\epsilon_{k}=+$ if $p_{k}$ is contained in $L$ and $\epsilon_{k}=-$ if $p_{k}$ is contained in $M$.

Theorem 2.2. Given a quasi-parabolic bundle $(E, \boldsymbol{p})$ over an elliptic curve $C$, the following conditions are equivalents

(1) $(E, \boldsymbol{p})$ is $\nu$-flat;

(2) every direct summand of $(E, \boldsymbol{p})$ is of parabolic degree zero with respect to $\nu$.

The proof will be given in Section 2.2.2. As a consequence, one obtains the following corollary for generic exponents $\nu$ satisfying the Fuchs Relation

$$
d+\sum_{k=1}^{n}\left(\nu_{k}^{+}+\nu_{k}^{-}\right)=0 .
$$

Corollary 2.3. Assume $\nu_{1}^{\epsilon_{1}}+\cdots+\nu_{n}^{\epsilon_{n}} \notin \mathbb{Z}$ for any $\epsilon_{k} \in\{+,-\}$. Given a quasiparabolic bundle $(E, \boldsymbol{p})$ over an elliptic curve $C$, with $\operatorname{deg}(E)=d$, the following conditions are equivalents

(1) $(E, \boldsymbol{p})$ is $\nu$-flat;

(2) $(E, \boldsymbol{p})$ is indecomposable.

Recall that over $\mathbb{P}^{1}$, the above conditions (1) and (2) are equivalent to $(E, \mathbf{p})$ be simple, i.e. the only automorphisms of $E$ preserving parabolics are scalar. Here, over an elliptic curve it is no more true. As in Remark 2.1. if $\mathbf{p}$ is a parabolic structure on $E_{0}$ with all parabolics lying on $\mathcal{O}_{C} \hookrightarrow E_{0}$ then $\operatorname{End}_{0}\left(E_{0}, \mathbf{p}\right)=\mathbb{C}$. Hence, $\left(E_{0}, \mathbf{p}\right)$ is not simple:

$$
\operatorname{Aut}\left(E_{0}, \mathbf{p}\right)=\left\{\left(\begin{array}{ll}
a & b \\
0 & a
\end{array}\right) ; a \in \mathbb{C}^{*}, \quad b \in \mathbb{C}\right\} .
$$

2.2.1. Preliminary lemmas. The easy part of Theorem 2.2 is $(1) \Rightarrow(2)$. As we shall see in the following lemma, it is a consequence of Fuchs Relation.

Lemma 2.4. If $(E, \boldsymbol{p})$ is $\nu$-flat, then every direct summand of $(E, \boldsymbol{p})$ is of parabolic degree zero.

Proof. Let us suppose $(E, \mathbf{p})=(L, \mathbf{q}) \oplus(M, \mathbf{r})$. The bundle $E$ is defined by gluing local charts $U_{i} \times \mathbb{C}^{2}$ with transition matrices

$$
M_{i j}=\left(\begin{array}{cc}
a_{i j} & 0 \\
0 & b_{i j}
\end{array}\right)
$$


where the subbundles $L$ and $M$ are respectively generated by $e_{1}$ and $e_{2}$.

Let $\nabla$ be a connection over $(E, \mathbf{p})$ with local exponent $\nu$, given in those charts by $\nabla=d+A_{i}$ where

$$
A_{i}=\left(\begin{array}{cc}
\alpha_{i} & \beta_{i} \\
\gamma_{i} & \delta_{i}
\end{array}\right)
$$

First we note that $\nabla_{1}:=\left.\nabla\right|_{L}=d+\alpha_{i}$ defines a connection over $L$. In fact, the compatibility conditions for $\nabla$ imply

$$
\alpha_{j}=\alpha_{i}+a_{i j}^{-1} \cdot d a_{i j} .
$$

Then under hypothesis of $(E, \mathbf{p})$ be decomposable one obtains

$$
\operatorname{Res}_{t_{k}}\left(\nabla_{1}\right)=\nu_{k}^{\epsilon_{k}}
$$

where $\epsilon_{k}=+$ if $p_{k}$ lies in $L$ and $\epsilon_{k}=-$ if $p_{k}$ lies in $M$. The conclusion of the proof follows from Fuchs Relation of $\nabla_{1}$ over $L$.

In order to prove that (2) implies (1) in Theorem 2.2, let us consider the set $\operatorname{End}_{0}(E, \mathbf{p})$ of traceless endomorphisms of $E$ leaving fixed the parabolics. In the context of $\mathfrak{s l}_{2}$-connections, the vanishing of this set is a sufficient condition to a quasi-parabolic vector bundle be $\nu$-flat. This has been proved in [1, Proposition 3] in the case $X=\mathbb{P}^{1}$, but the same proof works to the general case. Here we do the details for the reader's convenience.

Lemma 2.5. Let $C$ be a projective smooth curve of genus $g \geq 0$ and $E$ be a rank 2 vector bundle over $C$ with trivial determinant bundle. If

$$
\operatorname{End}_{0}(E, \boldsymbol{p})=\{0\}
$$

then $(E, \boldsymbol{p})$ is $\nu$-flat for any $\nu$ satisfying the Fuchs Relation.

Proof. To give a logarithmic connection $\nabla$ on $(E, \mathbf{p})$ with local exponent $\nu$ it is equivalent to give an $\mathfrak{s l}_{2}$-connection $\nabla \otimes \zeta$ on $(E, \mathbf{p})$ for suitable connection $\zeta$ on $\mathcal{O}_{C}$. Then let us assume $\nu=\left( \pm \nu_{1}, \ldots, \pm \nu_{n}\right)$.

We must construct a logarithmic connection $\nabla$ over $(E, \mathbf{p})$ with local exponent $\nu$ satisfying

$$
\operatorname{Res}_{t_{k}}(\nabla)\left(p_{k}\right)=\nu_{k} \cdot p_{k}
$$

for any $k=1, \ldots, n$. We can do it locally on $C$. The obstruction to global existence is measured by a certain cohomology group. In fact, let $\nabla_{i}$ be a connection over $\left.E\right|_{U_{i}}$ satisfying the desired condition for any $k=1, \ldots, n$ such that $t_{k} \in U_{i}$ for suitable open sets $U_{i} \subset C$ covering $C$. Note that $\theta_{i j}=\nabla_{i}-\nabla_{j}$ satisfies $\operatorname{Res}_{t_{k}}\left(\theta_{i j}\right)\left(p_{k}\right)=0$, by (2.3). Then the differences $\nabla_{i}-\nabla_{j}$ define a cocycle

$$
\left\{\nabla_{i}-\nabla_{j}\right\} \in \mathrm{H}^{1}(C, \mathcal{E})
$$

where

$$
\mathcal{E}=\left\{\theta \in \mathcal{E}_{n d_{0}}(E) \otimes \Omega_{C}^{1}(D) ; \operatorname{Res}_{t_{k}}(\theta)\left(p_{k}\right)=0\right\} .
$$

Hence the global existence is insured by the vanishing of this cohomology group. Here $\mathcal{E} n d_{0}(E)$ is the sheaf of traceless endomorphisms. We will prove that there is an isomorphism

$$
\mathrm{H}^{1}(C, \mathcal{E}) \simeq \operatorname{End}_{0}(E, \mathbf{p})^{*}
$$


In order to prove it, let us remark that, from Serre's duality theorem, $\mathrm{H}^{1}(C, \mathcal{E})$ is dual to $\mathrm{H}^{0}\left(C, \mathcal{E}^{*} \otimes \Omega_{C}^{1}\right)$. Let us consider the $\mathcal{O}_{C}$-bilinear symmetric map

$$
\begin{aligned}
{\mathcal{E} n d_{0}(E) \times \mathcal{E} n d_{0}(E)} & \longrightarrow \mathcal{O}_{C} . \\
(A, B) & \mapsto \operatorname{tr}(A \cdot B)
\end{aligned}
$$

It induces an isomorphism $\psi: \mathcal{E} n d_{0}(E) \longrightarrow \mathcal{E} n d_{0}(E)^{*}$. Let $\tilde{\mathcal{E}}$ be the subsheaf of $\mathcal{E} n d_{0}(E)$ defined by

$$
\tilde{\mathcal{E}}=\left\{\theta \in \mathcal{E} n d_{0}(E) ; \theta\left(p_{k}\right)=0\right\} .
$$

We note that $\mathcal{E}$ coincides with $\tilde{\mathcal{E}} \otimes \Omega_{C}^{1}(D)$. Since the image of $\tilde{\mathcal{E}}$ by $\psi$ is $\tilde{\mathcal{E}}^{*}$ one obtains

$$
\tilde{\mathcal{E}}^{*} \otimes \mathcal{O}_{C}(-D)=\left\{\alpha \in \mathcal{E} n d_{0}(E) ; \alpha\left(p_{k}\right) \subset p_{k}\right\} .
$$

This implies that

$$
\mathrm{H}^{0}\left(C, \tilde{\mathcal{E}}^{*} \otimes \mathcal{O}_{C}(-D)\right)=\operatorname{End}_{0}(E, \mathbf{p}) .
$$

and we get the desired isomorphism:

$$
\mathrm{H}^{1}(C, \mathcal{E}) \simeq \operatorname{End}_{0}(E, \mathbf{p})^{*} .
$$

Now we shall give the proof of Theorem 2.2 ,

2.2.2. Proof of Theorem 2.2. It follows from Lemma 2.4 that (1) implies (2). Now let $(E, \mathbf{p})$ be a quasi-parabolic bundle satisfying the hypothesis $(2)$ of the statement. First, let us assume that $(E, \mathbf{p})$ is decomposable:

$$
(E, \mathbf{p})=\left(L_{1}, \mathbf{p}_{1}\right) \oplus\left(L_{2}, \mathbf{p}_{2}\right) .
$$

Since $\left(L_{i}, \mathbf{p}_{i}\right)$ has parabolic degree zero for each $i \in\{1,2\}$, there is a logarithmic connection $\alpha_{i}$ on $L_{i}$, satisfying

$$
\operatorname{Res}_{t_{k}}\left(\alpha_{i}\right)=\nu_{k}^{\epsilon_{k}}
$$

where $\epsilon_{k}=+$ if $p_{k}$ lies in $L_{i}$ and $\epsilon_{k}=-$ if $p_{k}$ does not lie in $L_{i}$. They define a diagonal connection

$$
\nabla=\left(\begin{array}{cc}
\alpha_{1} & 0 \\
0 & \alpha_{2}
\end{array}\right)
$$

on $(E, \mathbf{p})$ with local exponent $\nu$.

Now let $(E, \mathbf{p})$ be indecomposable. After elementary transformations and twists, one can assume that $E$ has trivial determinant bundle. Besides that, it is enough to show the existence of an $\mathfrak{s l}_{2}$-connection on $(E, \mathbf{p})$ with local exponent $\nu=\left( \pm \nu_{1}, \ldots, \pm \nu_{n}\right)$. It follows from Proposition 4.5 that

$$
\operatorname{End}_{0}(E, \mathbf{p})=\{0\}
$$

unless $E$ equals $E_{0}$, up to elementary transformations and twists, and all the parabolics lie in the maximal subbundle $\mathcal{O}_{C} \hookrightarrow E_{0}$. Then, from Lemma 2.5 to conclude the proof of Theorem 2.2, it is enough to consider the case $E_{0}$ with all the parabolics in $\mathcal{O}_{C} \hookrightarrow E_{0}$. We shall do it in the following lemma.

Lemma 2.6. Let $\left(E_{0}, \boldsymbol{p}\right)$ be a quasi-parabolic bundle where all the parabolics lie in the maximal subbundle $\mathcal{O}_{C} \hookrightarrow E_{0}$. Then there exists an $\mathfrak{s l}_{2}$-connection on $\left(E_{0}, \boldsymbol{p}\right)$ with given local exponent $\left( \pm \nu_{1}, \ldots, \pm \nu_{n}\right)$. 
Proof. The idea of the proof is the following: $E_{0}$ can be obtained from $\mathcal{O}_{C} \oplus \mathcal{O}_{C}\left(t_{1}\right)$ by one negative elementary transformation at a direction $\left.q \subset \mathcal{O}_{C} \oplus \mathcal{O}_{C}\left(t_{1}\right)\right|_{t_{1}}$ which is not contained in $\left.\mathcal{O}_{C}\right|_{t_{1}}$ neither in $\left.\mathcal{O}_{C}\left(t_{1}\right)\right|_{t_{1}}$. The maximal subbundle $\mathcal{O}_{C} \hookrightarrow E_{0}$ corresponds to the maximal subbundle $\mathcal{O}_{C}\left(t_{1}\right) \hookrightarrow \mathcal{O}_{C} \oplus \mathcal{O}_{C}\left(t_{1}\right)$. Then we need to construct a logarithmic connection $\nabla$ on $\mathcal{O}_{C} \oplus \mathcal{O}_{C}\left(t_{1}\right)$ with poles at $D=t_{1}+\cdots+t_{n}$, satisfying the following conditions:

(1) $\operatorname{Res}_{t_{1}}(\nabla)$ has $-\nu_{1}$ and $\nu_{1}-1$ as eigenvalues, where $-\nu_{1}$ is associated with the eigenspace $q$ as above;

(2) $\operatorname{Res}_{t_{k}}(\nabla), k=2, \ldots, n$, has $\pm \nu_{k}$ as eigenvalues, where $\nu_{k}$ is associated with the eigenspace $\left.\mathcal{O}_{C}\left(t_{1}\right)\right|_{t_{k}}$.

We can assume that the vector bundle $\mathcal{O}_{C} \oplus \mathcal{O}_{C}\left(t_{1}\right)$ is defined by the cocycle

$$
G_{i j}=\left(\begin{array}{cc}
1 & 0 \\
0 & a_{i j}
\end{array}\right)
$$

where $\left\{a_{i j}\right\}$ defines the line bundle $\mathcal{O}_{C}\left(t_{1}\right)$.

Now, to give a logarithmic connection $\nabla$ on $\mathcal{O}_{C} \oplus \mathcal{O}_{C}\left(t_{1}\right)$ with poles at $D$ is equivalent to give $\nabla=d+A_{i}$ in charts $U_{i} \subset C$ where

$$
A_{i}=\left(\begin{array}{cc}
\alpha_{i} & \beta_{i} \\
\gamma_{i} & \delta_{i}
\end{array}\right) \in \operatorname{GL}_{2}\left(\Omega_{U_{i}}^{1}(D)\right)
$$

with $\alpha=\left\{\alpha_{i}\right\}, \beta=\left\{\beta_{i}\right\}, \gamma=\left\{\gamma_{i}\right\}$ and $\delta=\left\{\delta_{i}\right\}$ satisfying the compatibility conditions:

$$
A_{i} \cdot G_{i j}=G_{i j} \cdot A_{j}+d G_{i j} \cdot G_{i j}^{-1}
$$

on each intersection $U_{i} \cap U_{j}$. Equivalently,

$$
\begin{cases}d+\alpha & \text { is a connection on } \mathcal{O}_{C} \\ d+\delta & \text { is a connection on } \mathcal{O}_{C}\left(t_{1}\right) \\ \beta & \text { defines a global section }\left\{a_{i} \beta_{i}\right\} \in \mathrm{H}^{0}\left(C, \Omega_{C}^{1}\left(D-t_{1}\right)\right) \\ \gamma & \text { defines a global section }\left\{a_{i}^{-1} \gamma_{i}\right\} \in \mathrm{H}^{0}\left(C, \Omega_{C}^{1}\left(D+t_{1}\right)\right)\end{cases}
$$

where $a_{i j}=\frac{a_{i}}{a_{j}}$ is a meromorphic resolution of the cocycle, the vector $e_{1}$ generates the subbundle $\mathcal{O}_{C}$ and $e_{2}$ generates $\mathcal{O}_{C}\left(t_{1}\right)$ on each chart $U_{i} \subset C$.

In order to find $\alpha$ and $\delta$ we define $\lambda^{+}$and $\lambda^{-}$as

$$
\begin{aligned}
& \lambda^{+}=\nu_{2}+\cdots+\nu_{n} \\
& \lambda^{-}=-\nu_{2}-\cdots-\nu_{n}-1 .
\end{aligned}
$$

Let $d+\alpha$ be a logarithmic connection on $\mathcal{O}_{C}$ satisfying

$$
\operatorname{Res}_{t_{1}}(\alpha)=\lambda^{+} ; \operatorname{Res}_{t_{k}}(\alpha)=-\nu_{k}, k \geq 2
$$

and $d+\delta$ be a logarithmic connection on $\mathcal{O}_{C}\left(t_{1}\right)$ satisfying

$$
\operatorname{Res}_{t_{1}}(\delta)=\lambda^{-} ; \operatorname{Res}_{t_{k}}(\delta)=\nu_{k}, k \geq 2 .
$$

It remains to find $\beta$ and $\gamma$ subject to the conditions (1) and (2) above. Since residues of $\alpha$ and $\delta$ have already been chosen, condition (2) is equivalent to the vanishing of $\beta$ in $t_{k}$ for $k \geq 2$, that is, $\beta$ is induced by an element

$$
\beta^{\prime} \in \mathrm{H}^{0}\left(C, \Omega_{C}^{1}\left(D-t_{1}\right) \otimes \mathcal{O}_{C}\left(-t_{2}-\cdots-t_{n}\right)\right)=\mathrm{H}^{0}\left(C, \Omega_{C}^{1}\right) .
$$

In addition, as $C$ is an elliptic curve, we can assume $\beta^{\prime} \in \mathbb{C}$. Then we have one degree of freedom to choose $\beta$ satisfying condition (2), which is determined by its residue at $t_{1}$, still denoted by $\beta^{\prime}=\operatorname{Res}_{t_{1}}(\beta)$. 
The choice of $\gamma$ is independent of condition (2). For instance for each $k \geq 2$, $\operatorname{Res}_{t_{k}}(\gamma)$ determines the eigenvector associated with the eigenvalue $-\nu_{k}$. But we have to choose $\gamma$ satisfying condition (1). Given $\gamma \in \mathrm{H}^{0}\left(C, \Omega_{C}^{1}\left(D+t_{1}\right)\right)$ with residue $\gamma^{\prime}$ at $t_{1}$, one gets

$$
\operatorname{Res}_{t_{1}}(\nabla)=\left(\begin{array}{cc}
\lambda^{+} & \beta^{\prime} \\
\gamma^{\prime} & \lambda^{-}
\end{array}\right)
$$

Then saying that $-\nu_{1}$ and $\nu_{1}-1$ are eigenvalues of $\operatorname{Res}_{t_{1}}(\nabla)$ is equivalent to

$$
\lambda^{+} \lambda^{-}-\beta^{\prime} \gamma^{\prime}=\left(-\nu_{1}\right)\left(\nu_{1}-1\right)
$$

We leave the reader to verify that this last equality is equivalent to

$$
\beta^{\prime} \gamma^{\prime}=\left(\nu_{1}+\lambda^{+}\right) \cdot\left(\nu_{1}-\lambda^{+}-1\right) \text {. }
$$

On one hand, if the term in the right side is non zero, we can find $\beta^{\prime} \neq 0$ and $\gamma^{\prime} \neq 0$ satisfying this equality. In fact, $\gamma^{\prime} \neq 0$ if and only if $\gamma$ does not vanish at $t_{1}$. Then we need to find $\gamma \in \mathrm{H}^{0}\left(C, \Omega_{C}^{1}\left(D+t_{1}\right)\right)$ which is not contained in $\mathrm{H}^{0}\left(C, \Omega_{C}^{1}(D)\right)$. We can do it, because $C$ is elliptic, hence the linear system which corresponds to $\mathrm{H}^{0}\left(C, \Omega_{C}^{1}\left(D+t_{1}\right)\right)$ has no base points. On the other hand, if the term in the right side equals zero, we set

$$
\begin{cases}\beta^{\prime}=0, \gamma^{\prime} \neq 0 & \text { if } \nu_{1}+\lambda^{+}=0 \\ \beta^{\prime} \neq 0, \gamma^{\prime}=0 & \text { if } \nu_{1}-\lambda^{+}-1=0\end{cases}
$$

Finally, with these choices we see that the eigenspace associated to $-\nu_{1}$ is not contained in $\left.\mathcal{O}_{C}\right|_{t_{1}}$ neither in $\left.\mathcal{O}_{C}\left(t_{1}\right)\right|_{t_{1}}$.

2.3. Indecomposable quasi-parabolic bundles. In view of Corollary 2.3 it is interesting to characterize degree one indecomposable rank 2 quasi-parabolic bundles when there is at least one parabolic point. Fix $\operatorname{det}(E)=\mathcal{O}_{C}(w), w \in C$.

Proposition 2.7. Assume $n \geq 1$ and let $L$ be a degree $k$ line bundle over the elliptic curve $C$. If the parabolic bundle $\left(L \oplus L^{-1}(w), \boldsymbol{p}\right)$ is indecomposable then

$$
-n+1 \leq 2 k \leq n+1
$$

Proof. Firstly let us assume $2 k<-n+1$. Since $C$ is elliptic, it follows from Riemann-Roch theorem that

$$
\operatorname{dim} \mathrm{H}^{0}\left(C, \mathcal{O} \oplus L^{-2}(w)\right)=-2 k+2 .
$$

Then the family of embeddings $L \hookrightarrow L \oplus L^{-1}(w)$ is of dimension $-2 k+1$. Our hypothesis on $k$ implies that we can choose an embedding of $L$ containing any parabolic lying outside $L^{-1}(w)$. In this case our quasi-parabolic bundle would be decomposable. The same argument works with $L^{-1}(w)$ instead of $L$ if $2 k>$ $n+1$.

The case $n=2$ is of particular interest for us. Then we shall give a simple consequence of this proposition that will be useful in the sequel. The following proposition characterizes quasi-parabolic bundles $(E, \mathbf{p}), \mathbf{p}=\left\{p_{1}, p_{2}\right\}$, of rank 2 and determinant $\mathcal{O}_{C}(w)$ arising in our moduli space of connections.

Proposition 2.8. Let $(E, \boldsymbol{p}), \boldsymbol{p}=\left\{p_{1}, p_{2}\right\}$, be a rank 2 quasi-parabolic bundle over an elliptic curve $C$ with $\operatorname{det}(E)=\mathcal{O}_{C}(w)$. If $(E, \boldsymbol{p})$ is indecomposable then one of the following assertions hold true

(1) $E \simeq L \oplus L^{-1}(w)$ with $\operatorname{deg}(L)=0$; moreover, the parabolic structure satifies: 
- $p_{1}, p_{2}$ do not lie neither on $L^{-1}(w)$, and nor on the same embedding $L \hookrightarrow E$ (a codimension one condition);

- $L^{2}=\mathcal{O}_{C}\left(w-t_{k}\right)$ for some $k=1,2$, and $p_{k}$ lie outside of $L$ and $L^{-1}(w)$;

(2) $E=E_{1}$ is indecomposable, defined by the unique non trivial extension

$$
0 \longrightarrow \mathcal{O}_{C} \longrightarrow E_{1} \longrightarrow \mathcal{O}_{C}(w) \longrightarrow 0
$$

Proof. If $E$ is decomposable with $\operatorname{det}(E)=\mathcal{O}_{C}(w)$ then Proposition 2.7 implies $E \simeq L \oplus L^{-1}(w)$ with $\operatorname{deg}(L)$ equals 0 or 1 . If $\operatorname{deg}(L)=0$ we are done. If $\operatorname{deg}(L)=1$, then we set $M:=L^{-1}(w)$ to get $E \simeq M^{-1}(w) \oplus M$. Since $\operatorname{deg}(M)=0$, this gives the first assertion when $E$ is decomposable. Now any decomposition of $E$ is given by the unique destabilizing subsheaf $L^{-1}(w)$ together with any embedding $L \hookrightarrow E$. Such an embedding is given by a linear combination of the initial factor $L$ with a global section of $\operatorname{Hom}\left(L, L^{-1}(w)\right) \simeq L^{-2}(w)$, so that we get a one parameter family of possible decompositions. We note that all embedings coincide with $L$ exactly at $t \in C$ where $L^{-2}(w)=\mathcal{O}_{C}(t)$. If $t \neq t_{1}$, then there is a unique $L \subset E$ passing through $p_{1}$, provided that $p_{1} \not \subset L^{-1}(w)$; then a generic $p_{2}$, i.e. not belonging to either that $L$ or $L^{-1}(w)$ will be an obstruction to decomposablity. On the other hand, if $t=t_{1}$, and if $p_{1}$ is generic, then there is no $L$ passing through and $(E, \mathbf{p})$ is indecomposable. After reasoning similarly at $t_{2}$, one easily deduce that the only cases where we cannot find a decomposition of $E$ compatible with parabolics are those two cases listed in the statement.

If $E$ is indecomposable and $\operatorname{det}(E)=\mathcal{O}_{C}(w)$, then its well known that $E$ contains $\mathcal{O}_{C}$ as maximal subbundle. Moreover, there is only one indecomposable rank 2 bundle, up to isomorphism, having $\mathcal{O}_{C}(w)$ as determinant bundle (see for example [14, Lemma 4.4 and 4.5]).

\section{Moduli SPACE OF CONNECTIONS ON ELliptiC CURVES With TWO POLES}

Now we shall fix the data for our moduli space of logarithmic connections with two poles over an elliptic curve; see Section 4.5 for a more complete introduction to moduli spaces of connections. Let $C$ be an elliptic curve, for computation we assume that $C \subset \mathbb{P}^{2}$ is the smooth projective cubic curve

$$
z y^{2}=x(x-z)(x-\lambda z)
$$

with $\lambda \in \mathbb{C}, \lambda \neq 0,1$. Let us denote by $w_{\infty}=(0: 1: 0) \in C$ the identity with respect to the group structure, and

$$
w_{0}=(0: 0: 1), w_{1}=(1: 0: 1), w_{\lambda}=(\lambda: 0: 1)
$$

the 2 -torsion points.

Let $D=t_{1}+t_{2}$ be a reduced divisor on $C$, where $t_{2}=-t_{1}$ with respect to the group structure of $C$, i.e. defined by say $x=t$. We assume $t_{1} \neq t_{2}$, i.e. $x \neq 0,1, \lambda, \infty$. Let us fix local exponents $\nu=\left(\nu_{1}, \nu_{2}\right) \in \mathbb{C}^{2}$ and define eigenvalues

$$
\nu=\left(\nu_{1}^{ \pm}, \nu_{2}^{ \pm}\right):=\left( \pm \frac{\nu_{1}}{2}-\frac{1}{2}, \pm \frac{\nu_{2}}{2}\right) .
$$

therefore satisfying Fuchs Relation

$$
\nu_{1}^{+}+\nu_{1}^{-}+\nu_{2}^{+}+\nu_{2}^{-}+1=0 .
$$


To avoid dealing with reducible connections, we assume moreover a generic condition

$$
\nu_{1} \pm \nu_{2} \notin \mathbb{Z} \backslash 2 \mathbb{Z} \quad \text { (i.e. not an odd integer) }
$$

so that $\nu_{1}^{\epsilon_{1}}+\nu_{2}^{\epsilon_{2}} \notin \mathbb{Z}$ for any $\epsilon_{k} \in\{+,-\}$.

Fix $\zeta: \mathcal{O}_{C}\left(w_{\infty}\right) \longrightarrow \mathcal{O}_{C}\left(w_{\infty}\right) \otimes \Omega_{C}^{1}\left(t_{1}\right)$ any rank one logarithmic connection on $\mathcal{O}_{C}\left(w_{\infty}\right)$ satisfying

$$
\operatorname{Res}_{t_{1}}(\zeta)=-1
$$

Since local $\zeta$-horizontal sections have a simple zero at $t_{1}$, the invertible sheaf generated by these sections is $\mathcal{O}_{C}\left(w_{\infty}-t_{1}\right)$, and $\zeta$ corresponds to a holomorphic connection on this latter bundle. In particular, the monodromy of $\zeta$ must be non trivial.

We denote by $\operatorname{Con}^{\nu}(C, D)$ the moduli space of triples $(E, \nabla, \mathbf{p})$ where

(1) $(E, \mathbf{p})$ is a rank 2 quasi-parabolic vector bundle over $(C, D)$ having $\mathcal{O}_{C}\left(w_{\infty}\right)$ as determinant bundle;

(2) $\nabla: E \longrightarrow E \otimes \Omega_{C}^{1}(D)$ is a logarithmic connection on $E$ with polar divisor $D$, having $\nu$ as local exponents, $\operatorname{Res}_{t_{k}}(\nabla)$ acts on $p_{k}$ by multiplication by $\nu_{k}^{+}$and its trace is given by $\operatorname{tr}(\nabla)=\zeta$;

(3) two triples $(E, \nabla, \mathbf{p})$ and $\left(E^{\prime}, \nabla^{\prime}, \mathbf{p}^{\prime}\right)$ are equivalent when there is an isomorphism between quasi-parabolic bundles $(E, \mathbf{p})$ and $\left(E^{\prime}, \mathbf{p}^{\prime}\right)$ conjugating $\nabla$ and $\nabla^{\prime}$.

The reason why we have chosen $\mathcal{O}_{C}\left(w_{\infty}\right)$ instead of $\mathcal{O}_{C}$ as our fixed determinant bundle is the following: there is an open subset of $\operatorname{Con}^{\nu}(C, D)$ formed by triples $\left(E_{1}, \nabla, \mathbf{p}\right)$ where $E_{1}$ is the unique indecomposable rank 2 bundle over $C$, up to isomorphism, corresponding to the extension

$$
0 \longrightarrow \mathcal{O}_{C} \longrightarrow E_{1} \longrightarrow \mathcal{O}_{C}\left(w_{\infty}\right) \longrightarrow 0 .
$$

This allow us to avoid dealing with varrying underlying vector bundle. Some consequences of this choice will be more clear in the next section.

In the next lines, we will study the forgetful map Bun $:(E, \nabla, \mathbf{p}) \mapsto(E, \mathbf{p})$ from $\operatorname{Con}^{\nu}(C, D)$ to the moduli space $\operatorname{Bun}(C, D)$ of parabolic bundles $(E, \mathbf{p})$ having $\mathcal{O}_{C}\left(w_{\infty}\right)$ as determinant bundle. Actually, the construction of the moduli space $\operatorname{Con}^{\nu}(C, D)$ needs a choice of weights to impose a stability condition. But under our generic hypothesis on $\nu$, all connections are stable and the construction does not depend of this choice. On the other hand, to obtain a good moduli space of quasi-parabolic bundles, we need to introduce a stability condition (see Section 4.1).

3.1. Moduli space of parabolic vector bundles. In this section we recall the construction of the moduli space of parabolic bundles over an elliptic curve, see 6 for details. Given weights $\mu=\left(\mu_{1}, \mu_{2}\right) \in[0,1]^{2}$, let $\operatorname{Bun}_{w_{\infty}}^{\mu}(C, D)$ be the moduli space of $\mu$-semistable parabolic bundles with fixed determinant bundle $\mathcal{O}_{C}\left(w_{\infty}\right)$. The space of weights is divided in two chambers by a wall $\mu_{1}+\mu_{2}=1$. Inside each chamber any point in $\mathrm{Bun}_{w_{\infty}}^{\mu}(C, D)$ is represented by the same bundle and all $\mu$-semistable bundles are $\mu$-stable. Strictly $\mu$-semistable bundles only occurs along the wall. It follows from $[6$, Theorem $\mathrm{A}]$ that $\operatorname{Bun}_{w_{\infty}}^{\mu}(C, D)$ is isomorphic to $\mathbb{P}^{1} \times \mathbb{P}^{1}$ for any choice of weights. We recall briefly this construction in the next lines to the readers convenience. 
Firstly, we describe parabolic bundles inside the chambers, see also [6. Proposition 4.4]. Let us denote by $X_{<}=\operatorname{Bun}_{w_{\infty}}^{<}(C, D)$ the moduli space corresponding to the chamber $0<\mu_{1}+\mu_{2}<1$ and by $X_{>}=\operatorname{Bun}_{w_{\infty}}^{>}(C, D)$ the other moduli space corresponding to $1<\mu_{1}+\mu_{2}<2$. All the $\mu$-semistable parabolic bundles arising in $X_{<}$are of the form $\left(E_{1}, \mathbf{p}=\left\{p_{1}, p_{2}\right\}\right)$. Maximal subbundles of $E_{1}$ have degree zero, then any parabolic bundle in $X_{<}$is $\mu$-stable. Each parabolic bundle is completely determined by

$$
\left.\left(p_{1}, p_{2}\right) \in E_{1}\right|_{t_{1}} \times\left. E_{1}\right|_{t_{2}} \simeq \mathbb{P}^{1} \times \mathbb{P}^{1} .
$$

Then, we get the identification $X_{<} \simeq \mathbb{P}^{1} \times \mathbb{P}^{1}$. When $1<\mu<2$, parabolic bundles $\left(E_{1},\left\{p_{1}, p_{2}\right\}\right)$ having a degree zero line bundle $L \hookrightarrow E_{1}$ passing through the two parabolic directions became unstable, where $L$ is destabilizing:

$$
\operatorname{Stab}(L)=1-\mu_{1}-\mu_{2}<0 .
$$

But we need to add parabolic bundles of the following form

$$
\left(L \oplus L^{-1}\left(w_{\infty}\right),\left\{p_{1}, p_{2}\right\}\right), \operatorname{deg}(L)=0
$$

where no parabolic $p_{k}$ lie on $L^{-1}\left(w_{\infty}\right)$ and not all $p_{k}$ lie on the same embedding $L \hookrightarrow E$ (see Proposition 2.8). Since automorphisms group of $L \oplus L^{-1}\left(w_{\infty}\right)$ is two dimensional, all parabolic bundles

$$
\left(L \oplus L^{-1}\left(w_{\infty}\right),\left\{p_{1}, p_{2}\right\}\right)
$$

with $L$ fixed, represent the same element in $X_{>}$. Then one obtains an identification $X_{>} \simeq \mathbb{P}^{1} \times \mathbb{P}^{1}$.

When $\mu$ is inside the wall, the picture is described in [6. Proposition 4.1 and Theorem 4.2]. If $\mu_{1}+\mu_{2}=1$ with $\mu_{k} \neq 0$ for $k=1,2$, respective parabolic bundles in $\Gamma_{<}$and $\Gamma_{>}$are identified and parabolic bundles $\left(L \oplus L^{-1}\left(w_{\infty}\right), \mathbf{p}\right)$ with both direction on $L$ appear. But if $\mu_{k}=0$, then we also find bundles $L \oplus L^{-1}\left(w_{\infty}\right)$ with $p_{k}$ lying on $L^{-1}\left(w_{\infty}\right)$.

Let us identify $C$ with its Jacobian variety $\operatorname{Jac}(C)$ of degree zero line bundles

$$
\begin{aligned}
C & \longrightarrow \operatorname{Jac}(C) . \\
p & \mapsto \mathcal{O}_{C}\left(w_{\infty}-p\right)
\end{aligned}
$$

The locus $\Gamma$ of parabolic bundles inside $X_{>}$that became unstable when we change the chamber are parametrized by $C$

$$
\begin{aligned}
C \simeq \operatorname{Jac}(C) & \hookrightarrow \Gamma \subset X_{<} \\
L & \mapsto\left(E, \mathbf{p}_{L}\right)
\end{aligned}
$$

where $E=E_{1}$ and $\mathbf{p}_{L}=\left\{p_{1}^{L}, p_{2}^{L}\right\}$ corresponds to the parabolic directions inside $L$. Similarly, we still call $\Gamma$ the respective locus inside $X_{>}$parametrized by the following map

$$
\begin{aligned}
C \simeq \operatorname{Jac}(C) & \hookrightarrow \Gamma \subset X_{>} \\
L & \mapsto\left(E, \mathbf{p}_{L}\right)
\end{aligned}
$$

where $E=L \oplus L^{-1}\left(w_{\infty}\right)$, one of the parabolics lying in $L$ and the other one outside both $L$ and $L^{-1}\left(w_{\infty}\right)$. This locus $\Gamma$ corresponds to a curve of type $(2,2)$ parametrized by $C$.

The two charts $X_{<}$and $X_{>}$are identified outside $\Gamma$ and this provides a stratification of the coarse moduli space of simple parabolic bundles

$$
\operatorname{Bun}_{w_{\infty}}(C, D)=X_{<} \sqcup X_{>} .
$$


That is, simple parabolic bundles are parametrized by two copies of $\mathbb{P}^{1} \times \mathbb{P}^{1}$ identified outside a $(2,2)$ curve $\Gamma$. Now we would like to characterize indecomposable but not simple parabolic bundles, see also [6, Table 1].

Proposition 3.1. Let $(E, \boldsymbol{p})$ be an indecomposable parabolic bundle over $(C, D)$ having $\mathcal{O}_{C}\left(w_{\infty}\right)$ as determinant line bundle. If $(E, \boldsymbol{p})$ is not simple, then

$$
(E, \boldsymbol{p})=\left(L \oplus L^{-1}\left(w_{\infty}\right), \boldsymbol{p}\right)
$$

where $L^{2}=\mathcal{O}_{C}\left(w_{\infty}-t_{k}\right), k \in\{1,2\}$. Moreover, $p_{k}$ does not lie in $L$ neither in $L^{-1}\left(w_{\infty}\right)$ and the other parabolic lies in $L^{-1}\left(w_{\infty}\right)$.

Proof. If $(E, \mathbf{p})$ is indecomposable but not simple, then it follows from Proposition 2.8 that

$$
(E, \mathbf{p})=\left(L \oplus L^{-1}\left(w_{\infty}\right), \mathbf{p}\right), \operatorname{deg}(L)=0 .
$$

Since $(E, \mathbf{p})$ is not simple, at least one of the parabolics lies in $L^{-1}\left(w_{\infty}\right)$. Writing

$$
L^{2}=\mathcal{O}_{C}\left(w_{\infty}-w\right)
$$

for some $w \in C$, one obtains

$$
\mathbb{P} E=\mathbb{P}\left(\mathcal{O}_{C} \oplus \mathcal{O}_{C}(w)\right) .
$$

The family of sections corresponding to the family of embeddings $L \hookrightarrow E$ has a base point at the fiber over $w \in C$. Therefore if $w$ is distinct of $t_{k}, k=1,2$, we can choose an $L$ containing the parabolic outside $L^{-1}\left(w_{\infty}\right)$. In this situation, $(E, \mathbf{p})$ is decomposable. Now if $w=t_{k}$, then $p_{k}$ must be outside both $L$ and $L^{-1}\left(w_{\infty}\right)$, and the other parabolic must lie in $L^{-1}\left(w_{\infty}\right)$; indeed, otherwise $(E, \mathbf{p})$ would be decomposable (see Figure 1).

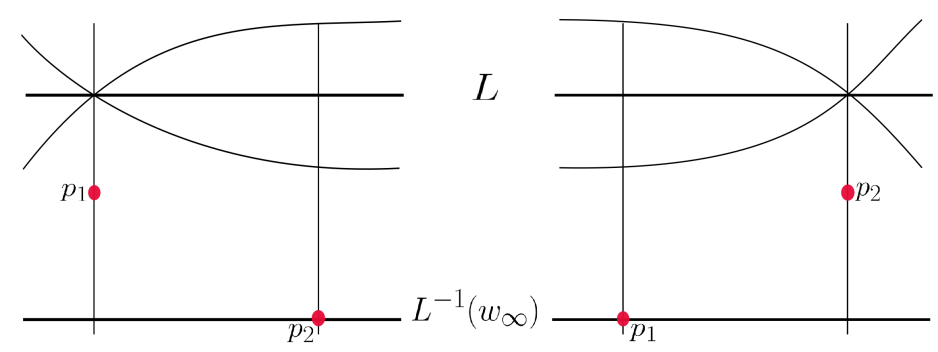

Figure 1. Indecomposable not simple, $L^{2}=\mathcal{O}_{C}\left(w_{\infty}-t_{k}\right), k \in\{1,2\}$.

3.2. Fiber compactification of the moduli space and Higgs fields. Let us consider the following open subset of the moduli space of connections corresponding to stable parabolic bundles in $X_{<}$:

$$
\operatorname{Con}_{<}^{\nu}(C, D)=\left\{(E, \nabla, \mathbf{p}) \in \operatorname{Con}^{\nu}(C, D) ; \quad(E, \mathbf{p}) \in X_{<}\right\} .
$$

Similarly, we define $\operatorname{Con}_{>}^{\nu}(C, D)$ with $X_{>}$instead of $X_{<}$. We note that $\operatorname{Con}_{<}^{\nu}(C, D)$ corresponds to triples $(E, \nabla, \mathbf{p})$ where $E=E_{1}$.

The union $\operatorname{Con}_{<}^{\nu}(C, D) \cup \operatorname{Con}_{>}^{\nu}(C, D)$ is the locus where the forgetful map with values at simple parabolic bundles $\operatorname{Con}^{\nu}(C, D) \longrightarrow \operatorname{Bun}_{w_{\infty}}(C, D)$ is well defined. 
Given a parabolic bundle $\left(E_{1}, \mathbf{p}\right) \in X_{<}$, any two connections $\nabla$ and $\nabla^{\prime}$ on it differ to each other by a parabolic Higgs field

$$
\nabla^{\prime}-\nabla=\Theta \in \mathrm{H}^{0}\left(C, \operatorname{End}\left(E_{1}, \mathbf{p}\right) \otimes \Omega_{C}^{1}(D)\right)=: \operatorname{Higgs}_{<}^{\nu}(C, D) .
$$

Since endomorphisms of $\left(E_{1}, \mathbf{p}\right)$ are scalars and $\Omega_{C}^{1} \simeq \mathcal{O}_{C}$, this vector space is two dimensional. Then, the fiber of the forgetful map Bun: $\operatorname{Con}_{<}^{\nu}(C, D) \longrightarrow X_{<}$over $\left(E_{1}, \mathbf{p}\right)$ identifies with the two dimensional affine space

$$
\operatorname{Bun}^{-1}\left(E_{1}, \mathbf{p}\right) \simeq \nabla^{0}+\mathrm{H}^{0}\left(C, \operatorname{End}\left(E_{1}, \mathbf{p}\right) \otimes \Omega_{C}^{1}(D)\right),
$$

where $\left(E_{1}, \nabla^{0}, \mathbf{p}\right)$ is any parabolic connection belonging to the fiber. We can compactify the affine $\mathbb{C}^{2}$-bundle Bun : $\operatorname{Con}_{<}^{\nu}(C, D) \longrightarrow X_{<}$by compactifying the fiber

$$
\overline{\operatorname{Bun}^{-1}\left(E_{1}, \mathbf{p}\right)}=\mathbb{P}\left(\mathbb{C} \cdot \nabla^{0} \oplus \mathrm{H}^{0}\left(C, \operatorname{End}\left(E_{1}, \mathbf{p}\right) \otimes \Omega_{C}^{1}(D)\right)\right) .
$$

Varying $\left(E_{1}, \mathbf{p}\right) \in X_{<}$and choosing a local section $\nabla^{0}$ over local open sets of $X_{<}$ we construct a $\mathbb{P}^{2}$-bundle

$$
\text { Bun }: \overline{\operatorname{Con}_{<}^{\nu}(C, D)} \longrightarrow X_{<} \simeq \mathbb{P}^{1} \times \mathbb{P}^{1}
$$

where

$$
\operatorname{PHiggs}_{<}^{\nu}(C, D):=\overline{\operatorname{Con}_{<}^{\nu}(C, D)} \backslash \operatorname{Con}_{<}^{\nu}(C, D)
$$

is the moduli space of projective Higgs fields.

3.3. From logarithmic connections to fuchsian systems with five poles. In order to study the $\mathbb{P}^{2}$-bundle

$$
\text { Bun }: \overline{\operatorname{Con}_{<}^{\nu}(C, D)} \longrightarrow X_{<}
$$

we perform three elementary transformations on $E_{1}$ to arrive on the trivial vector bundle. Then, we use global coordinates to obtain an explicit universal family for our moduli space. The idea is the following. For any triple

$$
(E, \nabla, \mathbf{p}) \in \overline{\operatorname{Con}_{<}^{\nu}(C, D)}
$$

the underlying rank 2 bundle is always $E=E_{1}$. It is well known that $E_{1}$ can be obtained from the trivial vector bundle $\mathcal{O}_{C} \oplus \mathcal{O}_{C}$ by three elementary transformations on distinct basis and distinct fibers (see for instance [14, Theorem 4.8]). Therefore, to give a logarithmic connection on $E_{1}$ is equivalent to give a fuchsian system on the trivial bundle having three apparent singular points. This will be explained in the next few lines.

We say that $t \in C$ is an apparent singular point for $\nabla$ if

(1) the residual part $\operatorname{Res}_{t} \nabla$ has $\left\{\frac{1}{2},-\frac{1}{2}\right\}$ as eigenvalues; and

(2) the $\frac{1}{2}$-eigenspace of $\operatorname{Res}_{t} \nabla$ is also invariant by the constant part of the connection matrix.

These conditions does not depend on the choice of local trivialization for $E$; condition (2) is equivalent to say that the local monodromy is semi-simple, i.e. $\pm I$.

Remark 3.2. We shall note that if $\nabla$ is an $\mathfrak{s l}_{2}$ connection, requiring that $t$ is an apparent singularity with a given direction $p$ imposes three linear conditions on the coefficients of $\nabla$. In fact, if we denote by $A_{-1}, A_{0} \in G L(2, \mathbb{C})$ the residual and the constant part of the connection matrix, respectively, then $(i)$ and (ii) above means

$$
\left\{\begin{array}{l}
\left(A_{-1}-\frac{1}{2} I\right) \cdot p=0 \\
\left(A_{0} \cdot p\right) \wedge p=0
\end{array}\right.
$$

in which gives us three linear conditions. 
We note that local monodromy does not change when we perform an elementary transformation over an apparent singular point, but the residual matrix becomes a multiple of the identity. In fact, let us assume that $x=t$ is an apparent singular point where the kernel of the residual part is

$$
\mathbf{p}=\left(\begin{array}{l}
1 \\
0
\end{array}\right)
$$

Then around $x=t$ the connection matrix writes

$$
\nabla=d+\left(\begin{array}{cc}
\frac{1}{2} & \beta_{-1} \\
0 & -\frac{1}{2}
\end{array}\right) \cdot \frac{d x}{(x-t)}+\left(\begin{array}{cc}
\alpha_{0} & \beta_{0} \\
0 & -\alpha_{0}
\end{array}\right) \cdot d x+o(x-t)
$$

Applying an elementary transformation

$$
\operatorname{elm}^{+}(Y)=\left(\begin{array}{cc}
x & 0 \\
0 & 1
\end{array}\right) \cdot Y
$$

we see that the residual matrix becomes

$$
\left(\begin{array}{cc}
-\frac{1}{2} & 0 \\
0 & -\frac{1}{2}
\end{array}\right)
$$

We now fix the data in order to define the moduli space of fuchsian systems with five poles on $C$ and three apparent singular points. Consider the 2-torsion points $w_{i}=(i, 0) \in C$, for $i=0,1, \lambda$, defined in (3.2). The divisor defined by them is linearly equivalent to $3 w_{\infty}$

$$
w_{0}+w_{1}+w_{\lambda} \sim 3 w_{\infty}
$$

Let us consider the reduced divisor

$$
D^{\prime}=w_{0}+w_{1}+w_{\lambda}+t_{1}+t_{2}
$$

and fix a local exponent

$$
\theta=\left( \pm \frac{1}{2}, \pm \frac{1}{2}, \pm \frac{1}{2}, \pm \frac{\nu_{1}}{2}, \pm \frac{\nu_{2}}{2}\right)
$$

We denote by $\operatorname{Syst}^{\theta}\left(C, D^{\prime}\right)$ the moduli space of $\mathfrak{s l}_{2}$-Fuchsian systems (i.e. $\log$ arithmic $\mathfrak{s l}_{2}$-connections on the trivial bundle) having $D^{\prime}$ as divisor of poles, $\theta$ as local exponents and such that

- the three singular points $w_{0}, w_{1}$ and $w_{\lambda}$ are apparent singular points;

- the corresponding $\frac{1}{2}$-eigenspaces $q_{i}$ are pairwise distinct.

In other words, up to isomorphism of the trivial vector bundle, one can assume $p_{i}=\left(w_{i},(i, 0)\right)$, for $i=0,1, \lambda$.

Proposition 3.3. There is an isomorphism of moduli spaces

$$
\operatorname{Syst}^{\theta}\left(C, D^{\prime}\right) \stackrel{\sim}{\longrightarrow} \operatorname{Con}_{<}^{\nu}(C, D)
$$

Proof. Let us describe the isomorphism (3.6); let $(E, \nabla, \mathbf{p})$ be a point in $\operatorname{Syst}^{\theta}\left(C, D^{\prime}\right)$. Consider the composition of three positive elementary transformations

$$
e l=e l e m_{w_{0}, w_{1}, w_{\lambda}}^{+}: \mathcal{O}_{C} \oplus \mathcal{O}_{C} \rightarrow-\rightarrow \tilde{E}
$$




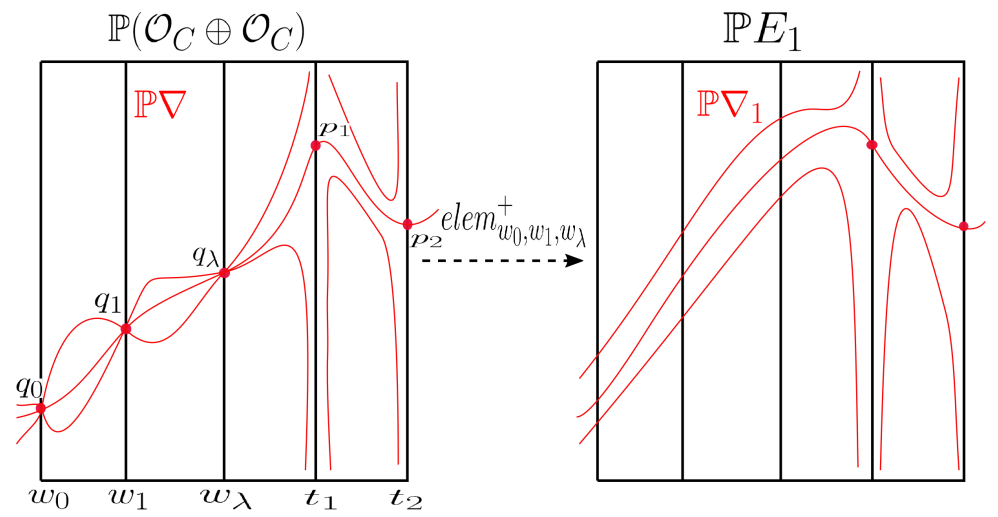

FIGURE 2. Elementary Transformation

on distinct basis points $\left\{w_{0}, w_{1}, w_{\lambda}\right\}$ and distinct fibers $\left\{q_{0}, q_{1}, q_{\lambda}\right\}$ (see Figure 22). After this birational modification of the trivial bundle, the corresponding indecomposable rank 2 vector bundle $\tilde{E}$ has determinant

$$
\operatorname{det} \tilde{E}=\mathcal{O}_{C}\left(w_{0}+w_{1}+w_{\lambda}\right) .
$$

Since $w_{0}+w_{1}+w_{\lambda}$ is linearly equivalent to $3 w_{\infty}$ then

$$
\operatorname{det} \tilde{E}=\mathcal{O}_{C}\left(3 w_{\infty}\right) \text {. }
$$

Applying such composition of elementary transformations (3.7) to $\left(\mathcal{O}_{C} \oplus \mathcal{O}_{C}, \nabla\right)$ one obtains a new pair $(\tilde{E}, \tilde{\nabla})=e l_{*}\left(\mathcal{O}_{C} \oplus \mathcal{O}_{C}, \nabla\right)$. The eigenvalues $\left\{\frac{1}{2},-\frac{1}{2}\right\}$ of $\nabla$ over $w_{i}$, are changed by

$$
\begin{aligned}
\tilde{\nu}_{i}^{+} & =\frac{1}{2}-1=-\frac{1}{2} \\
\tilde{\nu}_{i}^{-} & =-\frac{1}{2}
\end{aligned}
$$

and other eigenvalues $\left\{\frac{\nu_{i}}{2},-\frac{\nu_{i}}{2}\right\}$ over $t_{i}$, for $i=1,2$, are left unchanged.

Then the birational map el yields a new connection $\tilde{\nabla}$ on $\tilde{E}$

$$
\tilde{\nabla}: \tilde{E} \longrightarrow \tilde{E} \otimes \Omega_{C}^{1}\left(w_{0}+w_{1}+w_{\lambda}+t_{1}+t_{2}\right)
$$

with local exponents

$$
\tilde{\nu}=\left(\tilde{\nu}_{1}^{ \pm}=-\frac{1}{2}, \tilde{\nu}_{2}^{ \pm}=-\frac{1}{2}, \tilde{\nu}_{3}^{ \pm}=-\frac{1}{2}, \pm \frac{\nu_{1}}{2}, \pm \frac{\nu_{2}}{2}\right) .
$$

Since singularities over $w_{i}$ are apparent, we promptly deduce that $\tilde{\nabla}$ has local monodromy $-I d$ over them. That is, these singular points are projectively apparent. Its trace connection $\operatorname{tr}(\tilde{\nabla})$ is the unique rank one connection $d-\frac{d y}{y}$ on

$$
\mathcal{O}_{C}\left(w_{0}+w_{1}+w_{\lambda}\right)=\mathcal{O}_{C}\left(3 w_{\infty}\right) .
$$

with trivial monodromy, poles on $w_{0}, w_{1}, w_{\lambda}$ and exponents -1 . In order to restore a connection on $E_{1}$ without singularities over $w_{i}$, for $i=0,1, \lambda$, and with trace $\zeta$, we have to twist $(\tilde{E}, \tilde{\nabla})$ by a suitable logarithmic rank one connection $\xi$ on $\mathcal{O}_{C}\left(-w_{\infty}\right)$, 
namely a square root $\xi$ of

$$
\begin{aligned}
\left(\mathcal{O}_{C}\left(-w_{\infty}\right), \xi\right)^{\otimes 2} & :=\left(\mathcal{O}_{C}\left(w_{\infty}\right), \zeta\right) \otimes\left(\mathcal{O}_{C}\left(3 \omega_{\infty}\right), \zeta \otimes\left(d-\frac{d y}{y}\right)\right)^{\otimes(-1)} \\
& =\left(\mathcal{O}_{C}\left(-2 \omega_{\infty}\right), \zeta \otimes\left(d+\frac{d y}{y}\right)\right)
\end{aligned}
$$

where $\zeta$ is the fixed trace connection in the moduli space $\operatorname{Con}_{<}^{\nu}(C, D)$. The resulting logarithmic connection $\nabla_{1}=\tilde{\nabla} \otimes \xi$ on $E_{1}=\tilde{E} \otimes \mathcal{O}_{C}\left(-w_{\infty}\right)$ is nonsingular over $w_{i}$, for $i=0,1, \lambda$, and has eingenvalues $\left\{\frac{\nu_{1}}{2}-\frac{1}{2},-\frac{\nu_{1}}{2}-\frac{1}{2}\right\}$ over $t_{1}$ and $\left\{\frac{\nu_{2}}{2},-\frac{\nu_{2}}{2}\right\}$ over $t_{2}$. The parabolic structure $\mathbf{p}_{1}$ on $E_{1}$ over $D=t_{1}+t_{2}$ is defined by the image by $e l$ of the parabolic structure on $\mathcal{O}_{C} \oplus \mathcal{O}_{C}$ forgetting the directions over $w_{i}, i=0,1, \lambda$.

Then for each element $(E, \nabla, \mathbf{p})$ in $\operatorname{Syst}^{\theta}\left(C, D^{\prime}\right)$, we have associated an element $\left(E_{1}, \nabla_{1}, \mathbf{p}_{1}\right)$ in $\operatorname{Con}^{\nu}(C, D)$. This process can be reversed, and one gets an isomorphism

$$
\begin{aligned}
\operatorname{Syst}^{\theta}\left(C, D^{\prime}\right) & \stackrel{\sim}{\longrightarrow} \operatorname{Con}_{<}^{\nu}(C, D) \\
(E, \nabla, \mathbf{p}) & \mapsto\left(E_{1}, \nabla_{1}, \mathbf{p}_{1}\right)
\end{aligned}
$$

In fact, the inverse of transformation $E_{1} \rightarrow \mathcal{O}_{C} \oplus \mathcal{O}_{C}$ obtained by reversing (3.7) and (3.8) can be described as follows. Let $L_{i} \subset E_{1}$ denotes the unique embedding of $\mathcal{O}_{C}\left(w_{i}-w_{\infty}\right)$ for $i=0,1, \lambda$. Then, $L_{i}$ is transformed in $\mathcal{O}_{C} \oplus \mathcal{O}_{C}$ as the constant (trivial) subbundle generated by $(i, 0)$ for $i=0,1, \lambda$. This characterizes the inverse transformation.

Remark 3.4. As shown in Figure 3, the subbundles $L_{0}, L_{1}, L_{\lambda} \subset E_{1}$ previously defined correspond, after projectivization, to sections of $\mathbb{P} E_{1}$ having +1 self-intersection. We similarly define $L_{\infty} \subset E_{1}$ as the unique trivial subbundle $\mathcal{O}_{C} \hookrightarrow E_{1}$. Through the transformation $\mathcal{O}_{C} \oplus \mathcal{O}_{C} \rightarrow E_{1}$ obtained by composing birational modification $e l$ (3.7) and twist by (3.8), one easily check that the subbundle $L_{\infty} \simeq \mathcal{O}_{C}$ of $E_{1}$ corresponds to the subbundle $\mathcal{O}_{C}\left(-w_{0}-w_{1}-w_{\lambda}+w_{\infty}\right) \hookrightarrow \mathcal{O}_{C} \oplus \mathcal{O}_{C}$ generated by the rational section

$$
(x, y) \mapsto\left(\begin{array}{l}
1 \\
x
\end{array}\right) .
$$

This subbundle corresponds, after projectivization, to the unique section

$$
s: C \longrightarrow C \times \mathbb{P}^{1}
$$

having +4 self-intersection, passing through the points

$$
q_{i}=((i, 0),(1: i)), \quad i=0,1, \lambda
$$

and which is invariant under the hyperelliptic involution.

3.4. Universal family of fuchsian systems. As we have seen in Proposition 3.3 the open subset $\operatorname{Con}_{<}^{\nu}(C, D)$ of the moduli space $\operatorname{Con}^{\nu}(X, D)$ is isomorphic to the moduli space of fuchian systems $\operatorname{Syst}^{\theta}\left(C, D^{\prime}\right)$ with three apparent singularities over $w_{i}$ and local exponent $\theta$ as in (3.5). In this section, we exhibit an explicit universal family for $\operatorname{Syst}^{\theta}\left(C, D^{\prime}\right)$, when restricted to two suitable open sets of parabolic bundles. As a consequence, we determine the $\mathbb{P}^{2}$-bundle

$$
\text { Bun : } \overline{\operatorname{Con}_{<}^{\nu}(C, D)} \longrightarrow X_{<} \simeq \mathbb{P}^{1} \times \mathbb{P}^{1} \text {. }
$$




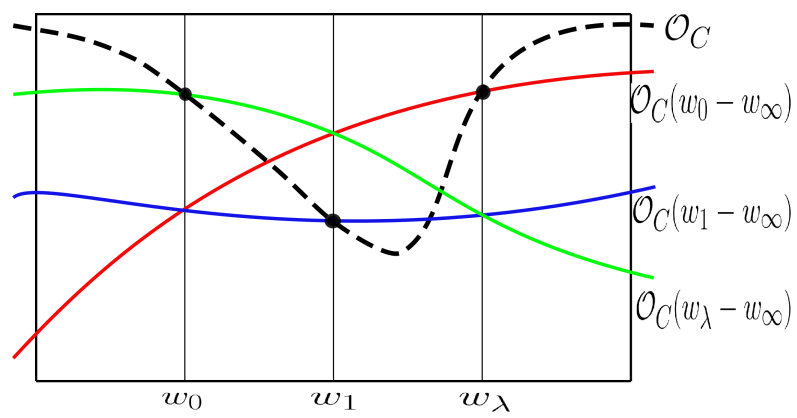

Figure 3 . Sections of $\mathbb{P} E_{1}$

Firstly we would like to write $X_{<} \simeq \mathbb{P}^{1} \times \mathbb{P}^{1}$, minus two points, as a union of two copies $U_{0} \cup U_{\infty}$ of $\mathbb{C}^{2}$ (see below). The $\mathbb{P}^{2}$-bundle above will be trivial when restricted to each one of them.

Let $U_{0}$ and $U_{\infty}$ be defined as follows

$$
\begin{aligned}
& U_{0}=\left\{\boldsymbol{z}=\left(\left(z_{1}: 1\right),\left(z_{2}: 1\right)\right) ; z_{1}, z_{2} \in \mathbb{C}\right\} \\
& U_{\infty}=\left\{\left(\left(1: Z_{1}\right),\left(1: Z_{2}\right)\right) ; Z_{1}, Z_{2} \in \mathbb{C}\right\} .
\end{aligned}
$$

Note that $U_{0} \cup U_{\infty}$ cover $\mathbb{P}^{1} \times \mathbb{P}^{1}$ minus two points:

$$
((1: 0),(0: 1)) \text { and }((0: 1),(1: 0)) \text {. }
$$

Each point $\boldsymbol{z} \in U_{0} \subset \mathbb{P}^{1} \times \mathbb{P}^{1}$ corresponds to a parabolic vector bundle $\left(E_{1}, \boldsymbol{z}\right)$ in $X_{<}$. By using the compositions of elementary transformations (3.7), one can identify each $\left(E_{1}, \boldsymbol{z}\right)$ in $X_{<}$with a parabolic bundle $\left(\mathcal{O}_{C} \oplus \mathcal{O}_{C}, \mathbf{p}_{\boldsymbol{z}}\right)$ where the parabolic structure is

$$
\mathbf{p}_{\boldsymbol{z}}:=\left\{q_{0}, q_{1}, q_{\lambda}, z_{1}, z_{2}\right\} .
$$

Thanks to Proposition 3.3. we identify the fiber $\operatorname{Bun}^{-1}\left(E_{1}, \boldsymbol{z}\right)$ with the space of fuchian systems in $\operatorname{Syst}^{\theta}\left(C, D^{\prime}\right)$ over the same fixed parabolic bundle

$$
\left(\mathcal{O}_{C} \oplus \mathcal{O}_{C}, \mathbf{p}_{\boldsymbol{z}}\right)
$$

for particular $\theta$ as in (3.5).

Any fuchsian system $\nabla \in \operatorname{Syst}^{\theta}\left(C, D^{\prime}\right)$ writes

$$
\nabla=d+\left(\begin{array}{cc}
\alpha & \beta \\
\gamma & -\alpha
\end{array}\right) \cdot \frac{d x}{y}
$$

where $\omega=d x / y$ is a global regular 1-form on $C$ and $\alpha, \beta, \gamma$ are rational functions on $C$ with at most simple poles on

$$
D^{\prime}=w_{0}+w_{1}+w_{\lambda}+t_{1}+t_{2} .
$$

The vector space that parametrizes the space of such rational functions is five dimension:

$$
\alpha, \beta, \gamma \in \mathrm{H}^{0}\left(C, \mathcal{O}_{C}\left(w_{0}+w_{1}+w_{\lambda}+t_{1}+t_{2}\right)\right) .
$$

Then, we have 15 parameters for $\alpha, \beta$ and $\gamma$. Each eigenvector $q_{i}$ over the apparent singular point $w_{i}$ imposes three linear conditions on the coefficients of $\nabla$, for each $i=0,1, \lambda$ (see Remark 3.2). Similarly, having $z_{1}$ (or $z_{2}$ ) as eigenvector with corresponding $\nu_{1}^{+}$(or $\nu_{2}^{+}$) as eigenvalue imposes two linear conditions. 


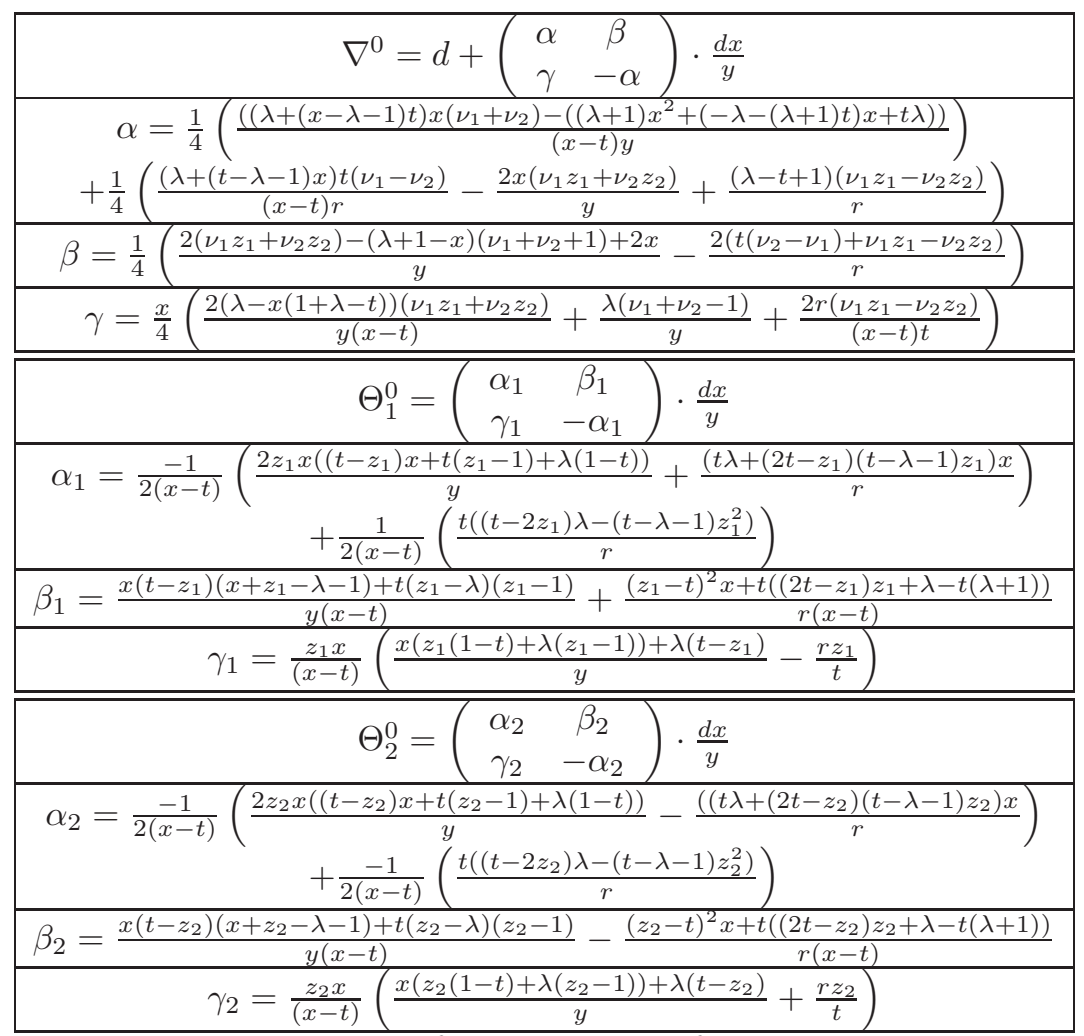

TABLE 1. Basis for the universal family over $U_{0}$

In order to find a basis for the moduli space of fuchsian systems $\nabla(\boldsymbol{z}) \in$ $\operatorname{Syst}^{\theta}\left(C, D^{\prime}\right)$ with given parabolic structure $\mathbf{p}_{\boldsymbol{z}}$, we have solved this linear system with 13 equations and 15 variables.

Let us denote by $t_{1}=(t, r)$ and $t_{2}=(t,-r)$. For any $\boldsymbol{z} \in U_{0}$, a fuchsian system $\nabla(\boldsymbol{z})$ like above writes

$$
\nabla(\boldsymbol{z})=\nabla^{0}(\boldsymbol{z})+c_{1} \cdot \Theta_{1}^{0}(\boldsymbol{z})+c_{2} \cdot \Theta_{2}^{0}(\boldsymbol{z})
$$

for $c_{1}, c_{2} \in \mathbb{C}$, where $\nabla^{0}(\boldsymbol{z})$ is a particular fuchsian system as well as $\Theta_{1}^{0}(\boldsymbol{z}), \Theta_{2}^{0}(\boldsymbol{z})$ are particular Higgs fields given by Table 1 .

In order to obtain also a universal family over the other open set $U_{\infty}$ of $X_{<}$, for each $\left(Z_{1}, Z_{2}\right)=\left(\frac{1}{z_{1}}, \frac{1}{z_{2}}\right) \in U_{\infty}$ the respective fuchsian and Higgs fields are

$$
\left\{\begin{array}{l}
\nabla^{\infty}\left(Z_{1}, Z_{2}\right)=\nabla^{0}\left(\frac{1}{Z_{1}}, \frac{1}{Z_{2}}\right)+\frac{\nu_{1}}{2} \cdot Z_{1} \cdot \Theta_{1}^{0}\left(\frac{1}{Z_{1}}, \frac{1}{Z_{2}}\right)+\frac{\nu_{2}}{2} \cdot Z_{2} \cdot \Theta_{2}^{0}\left(\frac{1}{Z_{1}}, \frac{1}{Z_{2}}\right) \\
\Theta_{1}^{\infty}\left(Z_{1}, Z_{2}\right)=\left(Z_{1}\right)^{2} \cdot \Theta_{1}^{0}\left(\frac{1}{Z_{1}}, \frac{1}{Z_{2}}\right) \\
\Theta_{2}^{\infty}\left(Z_{1}, Z_{2}\right)=\left(Z_{2}\right)^{2} \cdot \Theta_{2}^{0}\left(\frac{1}{Z_{1}}, \frac{1}{Z_{2}}\right)
\end{array}\right.
$$

Here we shall explain why the parameters $\nu_{1}, \nu_{2}$ appear in the definition of $\nabla^{\infty}$. Note that $\nabla^{0}$ is a rational section of $\operatorname{Con}_{<}{ }^{\nu}(C, D) \longrightarrow X_{<}$. In fact, $\nabla^{0}\left(\frac{1}{Z_{1}}, \frac{1}{Z_{2}}\right)$ has $\left\{Z_{1} Z_{2}=0\right\}$ as pole. This can be checked by using the explicit expression for $\nabla^{0}$ given in Table 1. Then the coeffients $\frac{\nu_{1}}{2}, \frac{\nu_{2}}{2}$ appearing in the definition of $\nabla^{\infty}$ have been chosen to make $\nabla^{\infty}$ regular. 
We summarize the above discussion in the next proposition. The intrinsic meaning of these basis will be given in the next section.

Proposition 3.5. For each $j \in\{0, \infty\}$, the $\mathbb{P}^{2}$-bundle Bun : $\overline{\operatorname{Con}_{<}^{\nu}(C, D)} \longrightarrow X_{<}$ is trivial when restricted to $U_{j}$ :

$$
\begin{aligned}
U_{j} \times \mathbb{P}^{2} & \stackrel{\simeq}{ }{\overline{\operatorname{Con}_{<}^{\nu}(C, D)}}_{U_{j}} \\
\left(u_{j},\left(C_{0}: C_{1}: C_{2}\right)\right) & \mapsto \mathbb{P}\left[C_{0} \cdot \nabla^{j}\left(u_{j}\right)+C_{1} \cdot \Theta_{1}^{j}\left(u_{j}\right)+C_{2} \cdot \Theta_{2}^{j}\left(u_{j}\right)\right]
\end{aligned}
$$

and the open set $\left.\operatorname{Con}_{<}^{\nu}(C, D)\right|_{U_{j}}$ is given by $\left(C_{0}: C_{1}: C_{2}\right)=\left(1: c_{1}: c_{2}\right)$.

3.5. An open set of the moduli space. Without loss of generality, we can assume that $\nu$ is as in (3.3)

$$
\left(\nu_{1}^{ \pm}, \nu_{2}^{ \pm}\right)=\left( \pm \frac{\nu_{1}}{2}-\frac{1}{2}, \pm \frac{\nu_{2}}{2}\right)
$$

(see Section 4.5). The condition $\nu_{k}^{+}=\nu_{k}^{-}$is equivalent to $\nu_{k}=0$ for each $k \in\{1,2\}$.

3.5.1. Description of $\overline{\mathrm{Con}_{<}^{\nu}(C, D)}$. We now apply results of Section 3.4 to study the $\mathbb{P}^{2}$-bundle

$$
\text { Bun }: \overline{\operatorname{Con}_{<}^{\nu}(C, D)} \longrightarrow X_{<} \simeq \mathbb{P}_{z_{1}}^{1} \times \mathbb{P}_{z_{2}}^{1}
$$

as well as the projectivized moduli space of Higgs fields

$$
\operatorname{PHiggs}_{<}^{\nu}(C, D):=\overline{\operatorname{Con}_{<}^{\nu}(C, D)} \backslash \operatorname{Con}_{<}^{\nu}(C, D) .
$$

Theorem 3.6. With identification $X_{<} \simeq \mathbb{P}_{z_{1}}^{1} \times \mathbb{P}_{z_{2}}^{1}$, the following assertions hold true:

(1) The canonical isomorphism $\operatorname{Higgs}_{<}^{\nu}(C, D) \stackrel{\sim}{\longrightarrow} T^{*} X_{<}$is given over charts $U_{0}$ and $U_{\infty}$ by the identifications

$$
\left\{\begin{array} { l l l } 
{ \Theta _ { 1 } ^ { 0 } } & { \mapsto } & { d z _ { 1 } } \\
{ \Theta _ { 2 } ^ { 0 } } & { \mapsto } & { d z _ { 2 } }
\end{array} \text { and } \left\{\begin{array}{lll}
\Theta_{1}^{\infty} & \mapsto & d Z_{1} \\
\Theta_{2}^{\infty} & \mapsto & d Z_{2}
\end{array}\right.\right.
$$

respectively. The Liouville form is $c_{1} d z_{1}+c_{2} d z_{2}$ and its differential

$$
\omega=d c_{1} \wedge d z_{1}+d c_{2} \wedge d z_{2}
$$

induces the canonical symplectic structure on $\left.\operatorname{Higgs}_{<}^{\nu}(C, D)\right|_{U_{0}}$.

(2) We have $\overline{\operatorname{Con}_{<}^{\nu}(C, D)}=\mathbb{P}\left(\mathcal{E}_{1}^{\nu}\right)$, where $\mathcal{E}_{1}^{\nu}$ is the extension of $\mathcal{O}_{X}$ by $T^{*} X_{<}$

$$
0 \longrightarrow T^{*} X_{<} \longrightarrow \mathcal{E}_{1}^{\nu} \longrightarrow \mathcal{O}_{X_{<}} \longrightarrow 0
$$

determined by

$$
\left(\frac{\nu_{1}}{2}, \frac{\nu_{2}}{2}\right) \in \mathrm{H}^{1}\left(X_{<}, T^{*} X_{<}\right) \simeq \mathrm{H}^{1}\left(\mathbb{P}_{z_{1}}^{1}, T^{*} \mathbb{P}_{z_{1}}^{1}\right) \oplus \mathrm{H}^{1}\left(\mathbb{P}_{z_{2}}^{1}, T^{*} \mathbb{P}_{z_{2}}^{1}\right) \simeq \mathbb{C}^{2}
$$

Proof. The isomorphism of (1) comes from Serre duality (see [7, Section 6]):

$$
\operatorname{Higgs}_{<}^{\nu}(C, D)=\mathrm{H}^{0}\left(C, \operatorname{End}\left(E_{1}, \mathbf{p}\right) \otimes \Omega_{C}^{1}(D)\right) \simeq \mathrm{H}^{1}\left(C, \operatorname{End}\left(E_{1}, \mathbf{p}\right)\right) \simeq T^{*} X_{<}
$$

and is well-known. By the same argument as in [7, Prop 6.1], we can check the identification (3.10) therefore proving (1).

It follows by Proposition 3.5 that $\left\{\nabla^{j}, \Theta_{1}^{j}, \Theta_{2}^{j}\right\}$, for each $j \in\{0, \infty\}$, are sections trivializing $\left.\overline{\operatorname{Con}_{<}^{\nu}(C, D)}\right|_{U_{j}}$

$$
\begin{aligned}
U_{j} \times \mathbb{P}^{2} & \stackrel{\simeq}{ }{\overline{\operatorname{Con}_{<}^{\nu}(C, D)}}_{U_{j}} \\
\left(u_{j},\left(C_{0}: C_{1}: C_{2}\right)\right) & \mapsto\left[C_{0} \cdot \nabla^{j}\left(u_{j}\right)+C_{1} \cdot \Theta_{1}^{j}\left(u_{j}\right)+C_{2} \cdot \Theta_{2}^{j}\left(u_{j}\right)\right] .
\end{aligned}
$$


By construction, in the intersection $U_{0} \cap U_{\infty}$ they satisfy

$$
\left\{\begin{array}{l}
\nabla^{\infty}=\nabla^{0}+\frac{\nu_{1}}{2} \cdot Z_{1} \cdot \Theta_{1}^{0}+\frac{\nu_{2}}{2} \cdot Z_{2} \cdot \Theta_{2}^{0} \\
\Theta_{1}^{\infty}=\left(Z_{1}\right)^{2} \cdot \Theta_{1}^{0} \\
\Theta_{2}^{\infty}=\left(Z_{2}\right)^{2} \cdot \Theta_{2}^{0}
\end{array}\right.
$$

Therefore, transition chart from $U_{\infty} \times \mathbb{C}^{3}$ to $U_{0} \times \mathbb{C}^{3}$ give us the following cocycle

$$
\left(\begin{array}{ccc}
1 & 0 & 0 \\
\frac{\nu_{1}}{2} \cdot Z_{1} & Z_{1}^{2} & 0 \\
\frac{\nu_{2}}{2} \cdot Z_{2} & 0 & Z_{2}^{2}
\end{array}\right)
$$

Since $U_{0} \cup U_{\infty}$ covers the base $X_{<} \simeq \mathbb{P}^{1} \times \mathbb{P}^{1}$ minus two points, this cocycle completely determines the extension. This proves that $\mathcal{E}_{1}^{\nu}$ corresponds to

$\left(\frac{\nu_{1}}{2}, \frac{\nu_{2}}{2}\right) \in \mathrm{H}^{1}\left(\mathbb{P}_{z_{1}}^{1} \times \mathbb{P}_{z_{2}}^{1}, T^{*}\left(\mathbb{P}_{z_{1}}^{1} \times \mathbb{P}_{z_{2}}^{1}\right)\right) \simeq \mathrm{H}^{1}\left(\mathbb{P}_{z_{1}}^{1}, T^{*} \mathbb{P}_{z_{1}}^{1}\right) \oplus \mathrm{H}^{1}\left(\mathbb{P}_{z_{2}}^{1}, T^{*} \mathbb{P}_{z_{2}}^{1}\right) \simeq \mathbb{C}^{2}$

This concludes item (2) of the statement.

We deduce the following corollary.

Corollary 3.7. The $\mathbb{P}^{2}$-bundle Bun $: \overline{\operatorname{Con}_{<}^{\nu}(C, D)} \longrightarrow X_{<}$is the trivial extension

$$
\overline{\operatorname{Con}_{<}^{\nu}(C, D)} \simeq \mathbb{P}\left(T^{*} X_{<} \oplus \mathcal{O}_{X_{<}}\right)
$$

if and only if $\nu_{1}=\nu_{2}=0$.

In fact, when $\nu_{1} \cdot \nu_{2} \neq 0$, then one easily see that all these $\mathbb{C}^{2}$-bundle are isomorphic between them, they correspond to the extension defined by the cocycle

$$
\left(\begin{array}{ccc}
1 & 0 & 0 \\
z_{1} & z_{1}^{2} & 0 \\
z_{2} & 0 & z_{2}^{2}
\end{array}\right)
$$

This will be done in a geometric way in the next section.

3.5.2. The moduli space $\operatorname{Con}_{<}^{\nu}(C, D)$ for $\nu_{1} \cdot \nu_{2} \neq 0$. When $\nu_{1} \cdot \nu_{2} \neq 0$ the kernel of the residual part of $\nabla \in \operatorname{Con}_{<}^{\nu}(C, D)$ over each $t_{i}$ has two distinct eigendirections

$$
\operatorname{ker}\left(\operatorname{Res}_{\mathrm{t}_{\mathrm{i}}} \nabla-\nu_{i}^{ \pm} I\right)=p_{i}^{ \pm}(\nabla) \in \mathbb{P}^{1}
$$

where $p_{i}^{\epsilon}$ corresponds to the eigenvalue $\nu_{i}^{\epsilon}$ for $\epsilon \in\{+,-\}$.

Let us denote by $\Delta \subset \mathbb{P}^{1} \times \mathbb{P}^{1}$ the diagonal and $S:=\left(\mathbb{P}^{1} \times \mathbb{P}^{1}\right) \backslash \Delta$ its complement. Then one gets a mapping which associates, to each $\nabla \in \operatorname{Con}_{<}^{\nu}(C, D)$, the eigenvectors of its residual part

$$
\begin{aligned}
\operatorname{Par}: \operatorname{Con}_{<}^{\nu}(C, D) & \longrightarrow S^{2} \\
\nabla & \mapsto\left(\left(p_{1}^{+}(\nabla), p_{1}^{-}(\nabla)\right),\left(p_{2}^{+}(\nabla), p_{2}^{-}(\nabla)\right)\right)
\end{aligned}
$$

In the next theorem, we will prove that this map is an isomorphism. For each $\epsilon \in\{+,-\}$, we shall denote by $\tau^{\epsilon}$ the projection corresponding to the directions $\left\{p_{1}^{\epsilon}, p_{2}^{\epsilon}\right\}$ :

$$
\begin{aligned}
\tau^{\epsilon}: S^{2} & \longrightarrow \mathbb{P}^{1} \times \mathbb{P}^{1} \\
\left(\left(p_{1}^{+}, p_{1}^{-}\right),\left(p_{2}^{+}, p_{2}^{-}\right)\right) & \mapsto\left(p_{1}^{\epsilon}, p_{2}^{\epsilon}\right)
\end{aligned}
$$


Similarly, for each $\epsilon \in\{+,-\}$, there is a projection $B u n^{\epsilon}$ corresponding to the respective parabolic structure associated to eigenvalues $\left\{\nu_{1}^{\epsilon}, \nu_{2}^{\epsilon}\right\}$.

$$
\begin{aligned}
\operatorname{Bun}^{\epsilon}: \mathrm{Con}_{<}^{\nu}(C, D) & \longrightarrow X_{<\cdot} \\
\left(E_{1}, \nabla, \mathbf{p}\right) & \mapsto\left(E_{1}, \mathbf{p}^{\epsilon}(\nabla)\right)
\end{aligned}
$$

Note that $\operatorname{Con}_{<}^{\nu}(C, D)$ has been defined by assuming that if $\left(E_{1}, \nabla, \mathbf{p}\right)$ is a point inside it then $\mathbf{p}$ corresponds to the eingenvalues $\left\{\nu_{1}^{+}, \nu_{2}^{+}\right\}$. This means that the above projection $\mathrm{Bun}^{+}$coincides with Bun of previous sections.

Theorem 3.8. If $\nu_{1} \cdot \nu_{2} \neq 0$ then $\mathrm{Par}: \operatorname{Con}_{<}^{\nu}(C, D) \longrightarrow S^{2}$ is an isomorphism between $\mathbb{C}^{2}$-affine bundles over $\mathbb{P}^{1} \times \mathbb{P}^{1}$

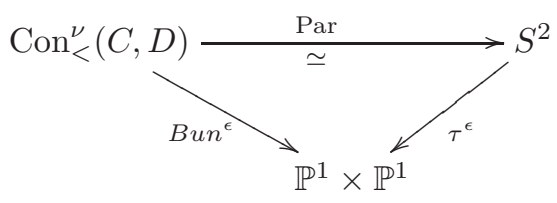

for each $\epsilon \in\{+,-\}$.

Proof. In order to prove the theorem we shall use the isomorphism

$$
\operatorname{Syst}^{\theta}\left(C, D^{\prime}\right) \simeq \operatorname{Con}_{<}^{\nu}(C, D)
$$

given by Proposition 3.3. We need to prove that the four eigenvectors $\left\{\mathbf{p}_{1}^{ \pm}(\nabla), \mathbf{p}_{2}^{ \pm}(\nabla)\right\}$ corresponding to the eigenvalues $\left\{ \pm \frac{\nu_{1}}{2}, \pm \frac{\nu_{2}}{2}\right\}$ determine the connection.

Since $\nu_{1} \cdot \nu_{2} \neq 0$, one has two distinct eigenvectors over each singular point $t_{1}$ and $t_{2}$. Then we can assume, without loss of generality, that $\mathbf{p}_{1}^{+}(\nabla)=(1: z)$ and $\mathbf{p}_{2}^{+}(\nabla)=(1: w)$. If $\mathbf{p}_{i}^{+}(\nabla)=(0: 1)$ for some $i=1,2$, the same argument works with $-\nu_{i}$ in place of $\nu_{i}$.

The fiber of

$$
\text { Bun : } \operatorname{Syst}^{\theta}\left(C, D^{\prime}\right) \longrightarrow X_{<} \simeq \mathbb{P}^{1} \times \mathbb{P}^{1}
$$

over $\boldsymbol{z} \in U_{0}$ is the two dimensional affine space

$$
\nabla_{\boldsymbol{c}}(\boldsymbol{z})=\nabla^{0}(\boldsymbol{z})+c_{1} \cdot \Theta_{1}^{0}(\boldsymbol{z})+c_{2} \cdot \Theta_{2}^{0}(\boldsymbol{z})
$$

varying $\boldsymbol{c}=\left(c_{1}, c_{2}\right) \in \mathbb{C}^{2}$, where $\nabla_{0}, \Theta_{1}^{0}$ and $\Theta_{2}^{0}$ were given in Table 1 Using the explicit expressions for them, we can compute the two eigenvectors of the kernel of $\nabla_{\boldsymbol{c}}(\boldsymbol{z})$ at each fiber over $t_{1}$ and $t_{2}$. They are

$$
\text { Par : } \nabla_{\boldsymbol{c}} \mapsto \begin{cases}p_{1}^{+}=\left(1: z_{1}\right) & p_{1}^{-}=\left(c_{1}: c_{1} z_{1}-\frac{\nu_{1}}{2}\right) \\ p_{2}^{+}=\left(1: z_{2}\right) & p_{2}^{-}=\left(c_{2}: c_{2} z_{2}-\frac{\nu_{2}}{2}\right)\end{cases}
$$

This proves that $\nabla_{\boldsymbol{c}}(\boldsymbol{z})$ is determined by its four residual eigendirections over $t_{1}$ and $t_{2}$.

Remark 3.9. The section $\nabla_{0}(\boldsymbol{z})$ of $B u n^{+}$is characterized by the property that

$$
\operatorname{Par}\left(\nabla_{0}\right)= \begin{cases}p_{1}^{+}=\left(1: z_{1}\right) & p_{1}^{-}=(0: 1) \\ p_{2}^{+}=\left(1: z_{2}\right) & p_{2}^{-}=(0: 1)\end{cases}
$$

It thus coincides with a fiber of $B u n^{-}$. 
Remark 3.10. Now we would like to remark that there is an isomorphism between $S^{2}$ and $\left(Q \times Q^{*}\right) \backslash \mathcal{I}$, where $Q \subset \mathbb{P}^{3}$ is the smooth quadric surface, $Q^{*}$ its dual variety and $\mathcal{I}$ is the incidence variety. As a consequence, we obtain the following isomorphism

$$
\operatorname{Con}_{<}^{\nu}(C, D) \simeq\left(Q \times Q^{*}\right) \backslash \mathcal{I} .
$$

In fact, let $\check{\mathbb{P}}^{3}$ be the set of hyperplanes in $\mathbb{P}^{3}$. The incidence variety $\mathcal{I}$ is defined as

$$
\mathcal{I}=\left\{\left(q, T_{r} Q\right) \in Q \times Q^{*} ; q \in T_{r} Q\right\} .
$$

We note that $Q$ and $Q^{*}$ are isomorphic. For instance, we can assume that $Q$ is defined by zero locus of $f:=x_{0} x_{3}-x_{1} x_{2}$. Its dual variety $Q^{*}$ can be identified with the image of the polar map $\phi_{f}: \mathbb{P}^{3} \rightarrow-\mathbb{P}^{3}$, which is defined by the derivatives of $f$. That is, $Q^{*}$ is just the image of $Q$ by a linear map and it is determined by the same equation $f$ as $Q$.

Via Segre embedding, we can identify $\left(Q \times Q^{*}\right) \backslash \mathcal{I}$ with $\left(\mathbb{P}^{1} \times \mathbb{P}^{1}\right)^{2} \backslash I$ where

$$
I=\left\{\left(\left(z_{1}, \zeta_{1}\right),\left(z_{2}, \zeta_{2}\right)\right) \in\left(\mathbb{P}^{1} \times \mathbb{P}^{1}\right)^{2} ; z_{1}=z_{2} \text { or } \zeta_{1}=\zeta_{2}\right\} .
$$

In addition, this last variety is isomorphic to $S^{2}$ :

$$
\begin{aligned}
\left(\mathbb{P}^{1} \times \mathbb{P}^{1}\right)^{2} \backslash I & \longrightarrow S^{2} \\
\left(\left(z_{1}, \zeta_{1}\right),\left(z_{2}, \zeta_{2}\right)\right) & \mapsto\left(\left(z_{1}, z_{2}\right),\left(\zeta_{1}, \zeta_{2}\right)\right) .
\end{aligned}
$$

3.6. Whole moduli space of connections. In order to describe the whole moduli space, we have to introduce other open charts.

3.6.1. Description of $\overline{\operatorname{Con}_{>}^{\nu}(C, D)}$. Recall that we have a stratification of the moduli space of simple parabolic bundles, see (3.4):

$$
\operatorname{Bun}_{w_{\infty}}(C, D)=X_{<} \sqcup X_{>} .
$$

Given $(E, \mathbf{p}) \in X_{>}$, we perform two elementary transformations elm $_{t_{1}, t_{2}}$ with center at $\mathbf{p}$, followed by a twisting by $\mathcal{O}_{C}\left(-w_{\infty}\right)$ in order to obtain a parabolic vector bundle $(\tilde{E}, \tilde{\mathbf{p}}) \in X_{<}$. Such operation defines a mapping $\phi_{D}$ from $X_{>}$to $X_{<}$that sends the locus $\Gamma \subset X_{>}$into the locus $\Gamma \subset X_{<}$. Identifying $X_{>}$and $X_{<}$with $\mathbb{P}^{1} \times \mathbb{P}^{1}$ we can show that this map corresponds to the following one

$$
\begin{aligned}
\phi_{D}: \mathbb{P}^{1} \times \mathbb{P}^{1} & \longrightarrow \mathbb{P}^{1} \times \mathbb{P}^{1} \\
\left(z_{1}, z_{2}\right) & \mapsto\left(z_{2}, z_{1}\right)
\end{aligned}
$$

(see [6, Proposition 5.5] for details).

Theorem 3.11. There is a fiber-preserving isomorphism $\Phi_{D}$ :

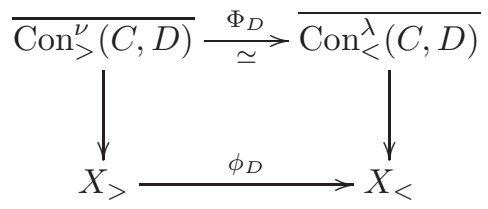

where $\lambda=\left(\lambda_{1}^{ \pm}, \lambda_{2}^{ \pm}\right)$with

$$
\left\{\begin{array}{l}
\lambda_{k}^{+}=\nu_{k}^{+}-1 / 2 \\
\lambda_{k}^{-}=\nu_{k}^{-}+1 / 2
\end{array}\right.
$$

for each $k \in\{1,2\}$. 
Proof. The above isomorphism is given by performing a positive elementary transformation over $D=t_{1}+t_{2}$. In fact, given $(E, \nabla, \mathbf{p}) \in \operatorname{Con}_{>}^{\nu}(C, D)$, let us consider the composition of two positive elementary transformations

$$
e l=e l e m_{t_{1}, t_{2}}^{+}: E \rightarrow E^{\prime}
$$

with center at $p_{1}^{+}$and $p_{2}^{+}$, respectively. The birational map el yields a new logarithmic connection $\nabla^{\prime}$ on $E^{\prime}$. We also shall fix a suitable rank one logarithmic connection on $\mathcal{O}_{C}\left(-w_{\infty}\right)$ with poles on $D$ satisfying the prescribed condition on residues:

$$
\operatorname{Res}_{t_{k}}(\zeta)=\frac{1}{2}
$$

for $k \in\{1,2\}$. Twisting $\left(E^{\prime}, \nabla^{\prime}\right)$ by $\left(\mathcal{O}_{C}\left(-w_{\infty}\right), \zeta\right)$ one obtains a pair

$$
(\tilde{E}, \tilde{\nabla})=\left(E^{\prime} \otimes \mathcal{O}_{C}\left(-w_{\infty}\right), \nabla^{\prime} \otimes \zeta\right)
$$

where $\tilde{E}$ has $\mathcal{O}_{C}\left(w_{\infty}\right)$ as determinant line bundle and the resulting connection $\tilde{\nabla}$ has local exponents $\lambda$ as stated.

Then we get a mapping $\Phi_{D}: \operatorname{Con}_{>}^{\nu}(C, D) \longrightarrow \operatorname{Con}_{<}^{\lambda}(C, D)$ in which can be reversed by the respective negative elementary transformation followed by a twisting by $\mathcal{O}_{C}\left(w_{\infty}\right)$.

Corollary 3.12. The $\mathbb{P}^{2}$-bundle Bun $: \overline{\operatorname{Con}_{>}^{\nu}(C, D)} \longrightarrow X_{>}$is the trivial extension

$$
\overline{\operatorname{Con}_{>}^{\nu}(C, D)}=\mathbb{P}\left(T^{*} X_{>} \oplus \mathcal{O}_{X_{>}}\right)
$$

if and only if $\nu_{k}^{+}-\nu_{k}^{-}=1$ for $k \in\{1,2\}$. If $\nu_{k}^{+}-\nu_{k}^{-} \neq 1$ for $k \in\{1,2\}$, then $\mathrm{Con}_{>}^{\nu}(C, D)$ is isomorphic to $S^{2}$.

Proof. First part of the statement is a consequence of Theorem 3.11 and Corollary 3.7. In the second part, we use Theorem 3.8 to conclude the proof.

3.6.2. Fiber over each indecomposable bundle. In view of Corollary 2.3, any parabolic bundle arising in our moduli space $\operatorname{Con}^{\nu}(C, D)$ is indecomposable. There are two types of indecomposable parabolic bundles: simple and not simple. As we have seen in Section 3.1 the moduli space of simple parabolic bundle having $\mathcal{O}_{C}\left(w_{\infty}\right)$ as determinant line bundle is a union of two copies of $\mathbb{P}^{1} \times \mathbb{P}^{1}$

$$
\operatorname{Bun}_{w_{\infty}}(C, D)=X_{<} \cup X_{>}
$$

identifying identical parabolic bundles outside a curve $\Gamma$ of type $(2,2)$. On the other hand, indecomposable not simple parabolic bundles were characterized in Proposition 3.1. There are eight different types: for each square root $L_{i, k}$ of $\mathcal{O}_{C}\left(w_{\infty}-t_{k}\right)$, $k \in\{1,2\}$ and $i \in\{1, \ldots, 4\}$, there is a unique parabolic bundle up to automorphism

$$
\mathcal{E}_{i, k}=\left(L_{i, k} \oplus L_{i, k}^{-1}\left(w_{\infty}\right), \mathbf{p}\right)
$$

with parabolic directions as in Proposition 3.1 (see Figure 1).

Let $\mathcal{C}_{\mathcal{E}} \subset \operatorname{Con}^{\nu}(C, D)$ be the set of $\nu$-flat connections over a given parabolic bundle $\mathcal{E}=(E, \mathbf{p})$. The whole moduli space $\operatorname{Con}^{\nu}(C, D)$ is the union of $\mathcal{C}_{\mathcal{E}}$ when $\mathcal{E}$ runs over all indecomposable parabolic bundles.

Proposition 3.13. Assume $\nu_{k}^{+} \neq \nu_{k}^{-}$for each $k=1,2$. The moduli space $\mathcal{C}_{\mathcal{E}}$ of $\nu$-flat connections over a given indecomposable parabolic bundle $\mathcal{E}=(E, \boldsymbol{p})$ is a two dimensional affine space. 
Proof. If $\mathcal{E}$ belongs to either $X_{<}$or $X_{>}$then $\mathcal{E}$ is simple. Since only automorphisms preserving parabolics are scalar, $\mathcal{C}_{\mathcal{E}}$ can be identified with the two dimensional affine space

$$
\mathcal{C}_{\mathcal{E}} \simeq \mathrm{H}^{0}\left(C, \text { End } \mathcal{E} \otimes \Omega_{C}^{1}(D)\right) .
$$

Now let $\mathcal{E}=\mathcal{E}_{i, k}$ as in (3.14) be an indecomposable parabolic bundle but not simple. For sake of simplicity, one may assume that $\mathcal{E}=\left(L \oplus L^{-1}\left(w_{\infty}\right)\right.$, p) with

$$
L^{2}=\mathcal{O}_{C}\left(w_{\infty}-t_{1}\right) .
$$

Where the parabolic direction $\mathbf{p}=\left(p_{1}, p_{2}\right)$ satisfies

$$
\left\{\begin{array}{l}
p_{1} \nsubseteq L_{t_{1}} \text { and } p_{1} \nsubseteq L^{-1}\left(w_{\infty}\right)_{t_{1}} \\
p_{2} \subset L^{-1}\left(w_{\infty}\right)_{t_{2}}
\end{array}\right.
$$

like Figure 1. By our hypothesis on $\nu$, any $\nu$-flat connection $\nabla \in \mathcal{C}_{\mathcal{E}}$ has two distinct eigendirections over each $t_{k}$, for each $k=1,2$

$$
\operatorname{Par}\left(\operatorname{Res}_{\mathrm{t}_{\mathrm{k}}} \nabla\right)=p_{k}^{ \pm}(\nabla) .
$$

Besides that, the "positive" directions coincide with $\mathbf{p}$, that is,

$$
\left\{\begin{array}{l}
p_{1}^{+}(\nabla)=p_{1} \\
p_{2}^{+}(\nabla)=p_{2} .
\end{array}\right.
$$

Also we note that since $p_{2} \subset L^{-1}\left(w_{\infty}\right)_{t_{2}}$ then $p_{2}^{-}(\nabla) \nsubseteq L^{-1}\left(w_{\infty}\right)_{t_{2}}$. We set

$$
q_{2}=p_{2}^{-}(\nabla)
$$

to simplify the notation. Therefore, each element $\nabla \in \mathcal{C}_{\mathcal{E}}$ defines a connection on $L \oplus L^{-1}\left(w_{\infty}\right)$ satisfying

$$
\left\{\begin{array}{l}
\operatorname{Res}_{t_{1}}(\nabla)\left(p_{1}\right)=\nu_{1}^{+} \cdot p_{1} \\
\operatorname{Res}_{t_{2}}(\nabla)\left(q_{2}\right)=\nu_{2}^{-} \cdot q_{2}
\end{array}\right.
$$

where

$$
\left\{\begin{array}{l}
p_{1} \nsubseteq L_{t_{1}} \text { and } p_{1} \nsubseteq L^{-1}\left(w_{\infty}\right)_{t_{1}} \\
q_{2} \nsubseteq \nsubseteq L^{-1}\left(w_{\infty}\right)_{t_{2}} .
\end{array}\right.
$$

A priori $q_{2}=p_{2}^{-}(\nabla)$ depends of $\nabla$. But since $p_{2}$ lies in the maximal subbundle $L^{-1}\left(w_{\infty}\right)$, the automorphism group of $E=L \oplus L^{-1}\left(w_{\infty}\right)$ fixing parabolic $\mathbf{p}=$ $\left\{p_{1}, p_{2}\right\}$ is two dimensional

$$
\operatorname{Aut}(E, \mathbf{p})=\left\{\left(\begin{array}{ll}
a & 0 \\
\gamma & a
\end{array}\right) ; a \in \mathbb{C}^{*}, \gamma \in \mathrm{H}^{0}\left(C, \mathcal{O}_{C}\left(t_{1}\right)\right)\right\} .
$$

Hence, we may suppose that all the connections $\nabla \in \mathcal{C}_{\mathcal{E}}$ have the same eigendirection $q_{2}$ outside $L^{-1}\left(w_{\infty}\right)$.

Reciprocally, given a connection $\nabla$ on $L \oplus L^{-1}\left(w_{\infty}\right)$ satisfying (3.15), we will show that $\nabla \in \mathcal{C}_{\mathcal{E}}$. In fact, suppose $\nabla$ is a connection on $\left(E,\left\{p_{1}, q_{2}\right\}\right)$ satisfying (3.15) and

$$
\left\{\begin{array}{l}
p_{1} \nsubseteq L_{t_{1}} \text { and } p_{1} \nsubseteq L^{-1}\left(w_{\infty}\right)_{t_{1}} \\
q_{2} \nsubseteq \nsubseteq L^{-1}\left(w_{\infty}\right)_{t_{2}}
\end{array}\right.
$$

We will prove that the second eigendirection of the residual part of $\nabla$ at $t_{2}$ which corresponds to $\nu_{2}^{+}$lies in $L^{-1}\left(w_{\infty}\right)$. In order to prove it, let us consider the apparent map with respect to $\mathcal{L}=L^{-1}\left(w_{\infty}\right)$

$$
\mathcal{L} \hookrightarrow E \stackrel{\nabla}{\longrightarrow} E \otimes \Omega_{C}^{1}\left(t_{1}+t_{2}\right) \longrightarrow(E / \mathcal{L}) \otimes \Omega_{C}^{1}\left(t_{1}+t_{2}\right)=L\left(t_{1}+t_{2}\right) .
$$


The zero divisor of the corresponding $\mathcal{O}_{C}$-linear map

$$
\varphi_{\nabla}: \mathcal{O}_{C} \longrightarrow \mathcal{L}^{-1} \otimes L\left(t_{1}+t_{2}\right)=\mathcal{O}_{C}\left(t_{2}\right)
$$

defines an element of the linear system $\mathbb{P H}^{0}\left(C, \mathcal{O}_{C}\left(t_{2}\right)\right)=\left|t_{2}\right|$. This means that its zero divisor is exactly $t_{2}$, because our curve is elliptic. Consequently, the residual part of $\nabla$ has an eigendirection, say $p_{2}$, lying in $L^{-1}\left(w_{\infty}\right)$. Since $q_{2}$ corresponds to eigenvalue $\nu_{2}^{-}$, then $p_{2}$ corresponds to $\nu_{2}^{+}$. It is enough to prove that $\nabla \in \mathcal{C}_{\mathcal{E}}$.

Let us denote by $\mathcal{E}_{-}$the parabolic bundle obtained by taking $q_{2}$ instead of $p_{2}$

$$
\mathcal{E}_{-}=\left(L \oplus L^{-1}\left(w_{\infty}\right),\left\{p_{1}, q_{2}\right\}\right)
$$

We have showed above that each $\nabla \in \mathcal{C}_{\mathcal{E}}$ can be seen as a connection on the parabolic bundle $\mathcal{E}_{-}$where

$$
\left\{\begin{array}{l}
\operatorname{Res}_{t_{1}}(\nabla)\left(p_{1}\right)=\nu_{1}^{+} \cdot p_{1} \\
\operatorname{Res}_{t_{2}}(\nabla)\left(q_{2}\right)=\nu_{2}^{-} \cdot q_{2}
\end{array}\right.
$$

and vice versa. Then $\mathcal{C}_{\mathcal{E}}$ can be identified with the affine vector space

$$
\mathcal{C}_{\mathcal{E}} \simeq \mathrm{H}^{0}\left(C, \operatorname{End}\left(\mathcal{E}_{-}\right) \otimes \Omega_{C}^{1}(D)\right) .
$$

As we know $\mathcal{E}_{-}$is simple, then the above cohomology group is a two dimensional vector space.

3.6.3. Patching open charts. Let us suppose $\nu_{k}^{+} \neq \nu_{k}^{-}$for each $k=1,2$. Given an element $(E, \nabla, \mathbf{p}) \in \operatorname{Con}^{\nu}(C, D)$, the parabolic direction $\mathbf{p}$ is nothing but the eigendirection for the residual part of $\nabla$ with respect to $\nu_{k}^{+}$. Under our hypothesis on $\nu$, the parabolic data is actually uniquely defined by the connection itself. Then we shall write just $(E, \nabla)$ instead of $(E, \nabla, \mathbf{p})$. Each $\nabla$ has two pairs of "positive" and "negative" eigendirections

$$
\left\{\begin{array}{l}
\mathbf{p}_{\nabla}^{+}=\left(p_{1}^{+}(\nabla), p_{2}^{+}(\nabla)\right) \\
\mathbf{p}_{\nabla}^{-}=\left(p_{1}^{-}(\nabla), p_{2}^{-}(\nabla)\right)
\end{array}\right.
$$

defined by the eigenvalues $\nu_{k}^{+}$and $\nu_{k}^{-}$, respectively.

The moduli space $\operatorname{Con}^{\nu}(C, D)$ admits two forgetful maps with values in the moduli space of simple parabolic bundles

$$
\begin{array}{rll}
\operatorname{Bun}^{+}: \operatorname{Con}^{\nu}(C, D) & \rightarrow & \operatorname{Bun}(C, D) \\
(E, \nabla) & \mapsto \quad\left(E, \mathbf{p}_{\nabla}^{+}\right)
\end{array}
$$

and

$$
\begin{array}{rll}
\operatorname{Bun}^{-}: \operatorname{Con}^{\nu}(C, D) & -\rightarrow & \operatorname{Bun}(C, D) . \\
(E, \nabla) & \mapsto & \left(E, \mathbf{p}_{\nabla}^{-}\right)
\end{array}
$$

They are complementary in the following sense. Indetermination points of Bun ${ }^{+}$ are not indetermination points of $B u n^{-}$and vice versa. Indeed, indetermination points of $\mathrm{Bun}^{+}$are of the form $(E, \nabla)$ such that $\left(E, \mathbf{p}_{\nabla}^{+}\right)$is not simple. There are eight indecomposable not simple parabolic bundles. It follows from Proposition 3.13 that the indetermination points of $\mathrm{Bun}^{+}$is a union of eight two dimensional affine spaces. But we could define our moduli space of connections by using $\nu_{k}^{-}$instead of $\nu_{k}^{+}$. This description for indetermination points of $\mathrm{Bun}^{+}$is completely symmetric for $B u n^{-}$. In fact, indetermination points of $B u n^{-}$are of the form $(E, \nabla)$ such that $\left(E, \mathbf{p}_{\nabla}^{-}\right)$is not simple. Besides that, there is an open set of $\operatorname{Con}^{\nu}(C, D)$ where fibers of $\mathrm{Bun}^{+}$and $\mathrm{Bun}^{-}$are transverse to each other. 
In order to describe our moduli space, one define the following open subsets

$$
\begin{aligned}
\mathcal{C} & :=\left\{\begin{array}{l}
\left.(E, \nabla) \in \operatorname{Con}^{\nu}(C, D) ;\left(E, \mathbf{p}_{\nabla}^{+}\right) \in X_{<}\right\} \\
(E, \nabla) \in \operatorname{Con}^{\nu}(C, D) ;\left(E, \mathbf{p}_{\nabla}^{-}\right) \in X_{<}
\end{array}\right. \\
& =\left\{\begin{array}{l}
\mathcal{C}_{>}^{+} \\
:=\left\{(E, \nabla) \in \operatorname{Con}^{\nu}(C, D) ;\left(E, \mathbf{p}_{\nabla}^{+}\right) \in X_{>}\right. \\
\mathcal{C}_{>}^{-}:=\left\{(E, \nabla) \in \operatorname{Con}^{\nu}(C, D) ;\left(E, \mathbf{p}_{\nabla}^{-}\right) \in X_{>}\right.
\end{array}\right\}
\end{aligned}
$$

Note that $\mathcal{C}=\operatorname{Con}_{<}^{\nu}(C, D)$ and $\mathcal{C}_{>}^{+}=\operatorname{Con}_{>}^{\nu}(C, D)$. The union $\mathcal{C} \cup \mathcal{C}_{>}^{+}$is the biggest open set where $\mathrm{Bun}^{+}$is well defined, i.e. its domain of definition is

$$
\operatorname{Dom}\left(\text { Bun }^{+}\right)=\mathcal{C} \cup \mathcal{C}_{>}^{+}
$$

We would like to remark that $\left(E, \mathbf{p}_{\nabla}^{+}\right) \in X_{<}$if, and only if $\left(E, \mathbf{p}_{\nabla}^{-}\right) \in X_{<}$, because $X_{<}$corresponds to parabolic bundles with underlying vector bundle $E=E_{1}$. Then $\mathcal{C} \cup \mathcal{C}_{>}^{-}$is the domain of definition of Bun $^{-}$

$$
\operatorname{Dom}\left(\text { Bun }^{-}\right)=\mathcal{C} \cup \mathcal{C}_{>}^{-} .
$$

Applying Corollary 3.12 with $\nu_{k}^{-}$in place of $\nu_{k}^{+}$, one obtains the same statement for $\mathcal{C}_{>}^{-}$instead of $\mathcal{C}_{>}^{+}$. Hence, Bun ${ }^{-}: \mathcal{C} \longrightarrow X_{>}$is an affine $\mathbb{C}^{2}$-bundle over $X_{>}$. We summarize the above discussion and previous results in the following theorem. We still denote by $\Delta \subset \mathbb{P}^{1} \times \mathbb{P}^{1}$ the diagonal and $S:=\left(\mathbb{P}^{1} \times \mathbb{P}^{1}\right) \backslash \Delta$ its complement.

Theorem 3.14. Assume $\nu_{1}^{\epsilon_{1}}+\nu_{2}^{\epsilon_{2}} \notin \mathbb{Z}$ for any $\epsilon_{k} \in\{+,-\}$. The moduli space $\operatorname{Con}^{\nu}(C, D)$ is a union of three affine $\mathbb{C}^{2}$-bundles over $\mathbb{P}^{1} \times \mathbb{P}^{1}$

$$
\operatorname{Con}^{\nu}(C, D)=\mathcal{C} \cup \mathcal{C}_{>}^{+} \cup \mathcal{C}_{>}^{-} \text {. }
$$

The intersection $\mathcal{U}:=\mathcal{C} \cap \mathcal{C}_{>}^{+} \cap \mathcal{C}_{>}^{-}$is the set of pairs $(E, \nabla)$ where $E=E_{1}$ and there is no degree zero subbundle of $E_{1}$ passing through neither $\boldsymbol{p}^{+}$nor $\boldsymbol{p}^{-}$. Moreover, there are fiber-preserving isomorphisms between affine $\mathbb{C}^{2}$-bundle over $\mathbb{P}^{1} \times \mathbb{P}^{1}$

$$
\Phi_{D}^{+}: \mathcal{C}_{>}^{+} \longrightarrow \operatorname{Con}_{<}^{\lambda}(C, D) \text { and } \Phi_{D}^{-}: \mathcal{C}_{>}^{-} \longrightarrow \operatorname{Con}_{<}^{\gamma}(C, D)
$$

where $\lambda=\left(\lambda_{1}^{ \pm}, \lambda_{2}^{ \pm}\right)$and $\gamma=\left(\gamma_{1}^{ \pm}, \gamma_{2}^{ \pm}\right)$satisfy

$$
\left\{\begin{array} { l } 
{ \lambda _ { k } ^ { + } = \nu _ { k } ^ { + } - 1 / 2 } \\
{ \lambda _ { k } ^ { - } = \nu _ { k } ^ { - } + 1 / 2 }
\end{array} \text { and } \left\{\begin{array}{l}
\gamma_{k}^{+}=\nu_{k}^{+}+1 / 2 \\
\gamma_{k}^{-}=\nu_{k}^{-}-1 / 2
\end{array}\right.\right.
$$

for each $k \in\{1,2\}$.

Proof. The hypothesis $\nu_{1}^{\epsilon_{1}}+\nu_{2}^{\epsilon_{2}} \notin \mathbb{Z}$ implies that only indecomposable parabolic bundles appears in our moduli space (see Corollary 2.3). If $(E, \nabla)$ does not belong to $\mathcal{C} \cup \mathcal{C}_{>}^{+}$, then $\left(E, \nabla, \mathbf{p}_{\nabla}^{+}\right)$is not simple. That is, $(E, \mathbf{p})=\mathcal{E}_{i, k}$ as in (3.14) and exactly one of the parabolics $p_{1}^{+}(\nabla)$ or $p_{2}^{+}(\nabla)$ lies in the maximal subbundle $L^{-1}\left(w_{\infty}\right)$ according with the square root $L_{i, k}$ of $\mathcal{O}_{C}\left(w_{\infty}-t_{i}\right)$ (see Figure 1). In particular, both parabolics $p_{1}^{-}(\nabla)$ and $p_{2}^{-}(\nabla)$ do not lie in $L^{-1}\left(w_{\infty}\right)$ and then

$$
\left(E,\left\{p_{1}^{-}(\nabla), p_{2}^{-}(\nabla)\right\}\right) \in X_{>} .
$$

This implies that $(E, \nabla) \in \mathcal{C}_{>}^{-}$and therefore one concludes that

$$
\operatorname{Con}^{\nu}(C, D)=\mathcal{C} \cup \mathcal{C}_{>}^{+} \cup \mathcal{C}_{>}^{-} .
$$

Now, let us describe the intersection $\mathcal{U}=\mathcal{C} \cap \mathcal{C}_{>}^{+} \cap \mathcal{C}_{>}^{-}$. If $(E, \nabla) \in \mathcal{C} \cap \mathcal{C}_{>}^{+}$, then $E=E_{1}$ and there is no degree zero line bundle passing through $\mathbf{p}_{\nabla}^{+}$, because

$$
\mathcal{C} \cap \mathcal{C}_{>}^{+}=\operatorname{Con}_{<}^{\nu}(C, D) \cap \operatorname{Con}_{>}^{\nu}(C, D) .
$$

The same conclusion can be done for $\mathbf{p}_{\nabla}^{-}$in place of $\mathbf{p}_{\nabla}^{+}$when $(E, \nabla) \in \mathcal{C}_{>}^{-}$. 
The fiber-preserving isomorphism $\Phi_{D}^{+}$is obtained by taking two positive elementary transformations with center at $\mathbf{p}_{\nabla}^{+}$followed by twisting by $\mathcal{O}_{C}\left(-w_{\infty}\right)$ (see Theorem 3.11 for details). The other fiber-preserving isomorphism $\Phi_{D}^{-}$is defined similarly taking the elementary transformation at $\mathbf{p}_{\nabla}^{-}$instead of $\mathbf{p}_{\nabla}^{+}$.

Recall that $S=\left(\mathbb{P}^{1} \times \mathbb{P}^{1}\right) \backslash \Delta$ and there are projections

$$
\begin{aligned}
\tau^{\epsilon}: S^{2} & \longrightarrow \mathbb{P}^{1} \times \mathbb{P}^{1} \\
\left(\left(p_{1}^{+}, p_{1}^{-}\right),\left(p_{2}^{+}, p_{2}^{-}\right)\right) & \mapsto\left(p_{1}^{\epsilon}, p_{2}^{\epsilon}\right)
\end{aligned}
$$

for $\epsilon \in\{+,-\}$, making $S^{2}$ a double $\mathbb{C}^{2}$-affine bundle over $\mathbb{P}^{1} \times \mathbb{P}^{1}$. Fibers of $\tau^{+}$ and $\tau^{-}$are transverse between them.

Theorem 3.15. Assume $\nu_{1}^{\epsilon_{1}}+\nu_{2}^{\epsilon_{2}} \notin \mathbb{Z}$ for any $\epsilon_{k} \in\{+,-\}$. If $\nu_{k}^{+}-\nu_{k}^{-} \notin\{0,1,-1\}$ for $k \in\{1,2\}$, then the moduli space $\operatorname{Con}^{\nu}(C, D)$ of $\nu$-flat connections over the elliptic curve $C$, minus two points, is a union of three copies of $S^{2}$

$$
\operatorname{Con}^{\nu}(C, D)=S^{2} \cup_{\Psi^{+}} S^{2} \cup_{\Psi^{-}} S^{2} .
$$

Where $\Psi^{ \pm}: S^{2} \rightarrow S^{2}$ are fiber-preserving isomorphisms outside a $(2,2)$ curve $\Gamma \subset \mathbb{P}^{1} \times \mathbb{P}^{1}$ isomorphic to $C$

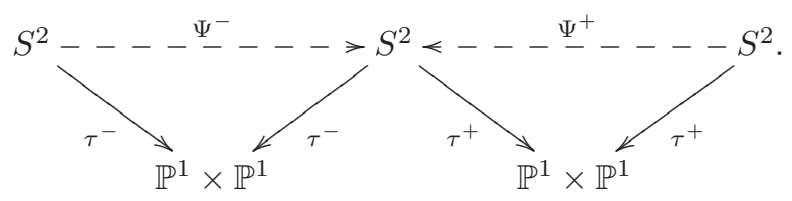

Proof. The three copies of $S^{2}$ are given by Theorem 3.6

$$
\operatorname{Con}^{\nu}(C, D)=\mathcal{C} \cup \mathcal{C}_{>}^{+} \cup \mathcal{C}_{>}^{+}
$$

In fact, $\nu_{k}^{+}-\nu_{k}^{-} \neq 0$ implies that $\mathcal{C} \simeq S^{2}$ (see Theorem 3.8 ). If $\nu_{k}^{+}-\nu_{k}^{-} \notin\{1,-1\}$, by the same reason, the two moduli spaces $\operatorname{Con}_{<}^{\gamma}(C, D)$ and $\operatorname{Con}_{<}^{\lambda}(C, D)$ of Theorem 3.6. can be also identified with $S^{2}$. Then we get the following fiber-preserving isomorphisms

$$
\Phi_{D}^{+}: \mathcal{C}_{>}^{+} \longrightarrow \operatorname{Con}_{<}^{\gamma}(C, D) \simeq S^{2} \text { and } \Phi_{D}^{-}: \mathcal{C}_{>}^{-} \longrightarrow \operatorname{Con}_{<}^{\lambda}(C, D) \simeq S^{2} .
$$

We define $\Psi^{+}$and $\Psi^{-}$as inverse of $\Phi_{D}^{+}$and $\Phi_{D}^{-}$, respectively. To conclude the proof of theorem we remark that $\mathcal{C}_{>}^{+}=\operatorname{Con}_{>}^{\nu}(C, D)$ intersect $\mathcal{C}=\operatorname{Con}_{<}^{\nu}(C, D)$ outside the locus of pairs $\left(E_{1}, \nabla\right)$ such that the respective parabolic bundle $\left(E_{1}, \mathbf{p}_{\nabla}^{+}\right)$has two directions $\mathbf{p}_{\nabla}^{+}=\left\{p_{1}^{+}, p_{2}^{+}\right\}$lying in the same degree zero line bundle $L \in \operatorname{Jac}^{0}(C) \simeq$ $C$. This locus is a $(2,2)$ curve $\Gamma \subset \mathbb{P}^{1} \times \mathbb{P}^{1}$ parametrized by $C$. The same argument works for $\mathcal{C}_{>}^{-}$with $\mathbf{p}_{\nabla}^{-}$instead of $\mathbf{p}_{\nabla}^{+}$(see Figure 4 ).

3.7. Apparent singularities. In this section, we will study the apparent map with respect to $\mathcal{O}_{C} \hookrightarrow E_{1}$ (see [13]). Each connection $\nabla$ on $E_{1}$ defines an $\mathcal{O}_{C}$-linear map:

$$
\mathcal{O}_{C} \stackrel{\iota}{\hookrightarrow} E_{1} \stackrel{\nabla}{\longrightarrow} E_{1} \otimes \Omega_{C}^{1}(D) \longrightarrow\left(E_{1} / \mathcal{O}_{C}\right) \otimes \Omega_{C}^{1}(D)
$$

where the last arrow is defined by the quotient map from $E_{1}$ to $E_{1} / \iota\left(\mathcal{O}_{C}\right)$ (denoted $\left.E_{1} / \mathcal{O}_{C}\right)$ for short. That is, we shall consider the mapping

$$
\varphi_{\nabla}: \mathcal{O}_{C} \longrightarrow\left(E_{1} / \mathcal{O}_{C}\right) \otimes \Omega_{C}^{1}(D)
$$




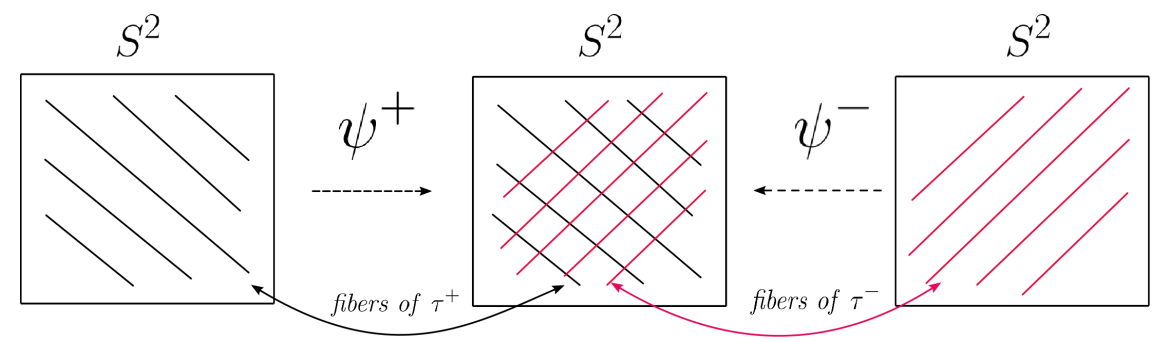

Figure 4. Description of the moduli space

Its zero divisor defines an element of the linear system

$$
\mathbb{P H}^{0}\left(C,\left(E_{1} / \mathcal{O}_{C}\right) \otimes \Omega_{C}^{1}(D)\right) .
$$

Since $D=t_{1}+t_{2}, \operatorname{det}\left(E_{1}\right)=\mathcal{O}_{C}\left(w_{\infty}\right)$ and $\Omega_{C}^{1} \simeq \mathcal{O}_{C}$ then

$$
\mathbb{P H}^{0}\left(C,\left(E_{1} / \mathcal{O}_{C}\right) \otimes \Omega_{C}^{1}(D)\right)=\left|\mathcal{O}_{C}\left(w_{\infty}+t_{1}+t_{2}\right)\right| \simeq \mathbb{P}^{2} .
$$

Then one gets a map

$$
A p p: \operatorname{Con}_{<}^{\nu}(C, D) \longrightarrow\left|\mathcal{O}_{C}\left(w_{\infty}+t_{1}+t_{2}\right)\right| \simeq \mathbb{P}^{2} .
$$

On the other hand, we have a mapping

$$
\text { Bun : } \operatorname{Con}_{<}^{\nu}(C, D) \longrightarrow X_{<} \simeq \mathbb{P}_{z_{1}}^{1} \times \mathbb{P}_{z_{2}}^{1}
$$

which comes from the forgetful map.

Definition 3.16. Let $V \simeq \mathbb{C}^{2} \subset X_{<} \simeq \mathbb{P}^{1} \times \mathbb{P}^{1}$ be the open subset corresponding to parabolic bundles $\left(E_{1}, \mathbf{p}\right), \mathbf{p}=\left\{p_{1}, p_{2}\right\}$, such that $p_{i} \neq(1: t)$ for $i=1,2$, where $t \in \mathbb{C}$ is the first coordinate of $t_{1}=(t, r) \in C$. We have the decomposition:

$$
\mathbb{P}_{z_{1}}^{1} \times \mathbb{P}_{z_{2}}^{1}=V \sqcup\left(\left\{z_{1}=t\right\} \cup\left\{z_{2}=t\right\}\right) \quad \text { and } \quad\left\{z_{1}=t\right\} \cap\left\{z_{2}=t\right\}=:\left\{u_{t}\right\} .
$$

Theorem 3.17. If $\nu_{1}+\nu_{2}+1 \neq 0$, then the map Bun $\times$ App defines a birational map

$$
\text { Bun } \times \text { App : } \overline{\operatorname{Con}_{<}^{\nu}(C, D)} \longrightarrow\left(\mathbb{P}_{z_{1}}^{1} \times \mathbb{P}_{z_{2}}^{1}\right) \times \mathbb{P}^{2}
$$

between $\mathbb{P}^{2}$-bundles over $\mathbb{P}_{z_{1}}^{1} \times \mathbb{P}_{z_{2}}^{1}$. Moreover, the following assertions hold true

(1) its restriction to $V \subset \mathbb{P}_{z_{1}}^{1} \times \mathbb{P}_{z_{2}}^{1}$ is an isomorphism

$$
\text { Bun } \times\left. A p p\right|_{B^{-1}(V)}: \operatorname{Bun}^{-1}(V) \stackrel{\simeq}{\longrightarrow} V \times \mathbb{P}^{2} ;
$$

(2) if $u \in\left\{z_{1}=t\right\} \cup\left\{z_{2}=t\right\} \backslash\left\{u_{t}\right\}$, then the image of

$$
\text { Bun } \times\left. A p p\right|_{B u n^{-1}(u)}: \mathbb{P}^{2} \longrightarrow \mathbb{P}^{2}
$$

is a line;

(3) if $\nu_{1}+\nu_{2}-1 \neq 0$ and $u=u_{t}$, then Bun $\times\left. A p p\right|_{B u n^{-1}\left(u_{t}\right)}$ is constant and its image is the point

$$
w_{\infty}+t_{1}+t_{2} \in\left|\mathcal{O}_{C}\left(w_{\infty}+t_{1}+t_{2}\right)\right| \simeq \mathbb{P}^{2} ;
$$

(4) if $\nu_{1}+\nu_{2}-1=0$ and $u=u_{t}$, then Bun ${ }^{-1}\left(u_{t}\right)$ lies in the indetermination locus of Bun $\times$ App.

Remark 3.18. A phenomenon similar to case (3) was observed in the genus zero case by Szilárd Szabó in [20]. 
Proof. Firstly, let us fix $\boldsymbol{z} \in U_{0} \subset X_{<}$. We will use the explicit basis $\left\{\nabla^{0}, \Theta_{1}^{0}, \Theta_{2}^{0}\right\}$ given in Table 1 to compute the mapping

$$
\operatorname{App}_{(\boldsymbol{z})}: \operatorname{Bun}^{-1}(\boldsymbol{z}) \simeq \mathbb{P}^{2} \longrightarrow\left|\mathcal{O}_{C}\left(w_{\infty}+t_{1}+t_{2}\right)\right| \simeq \mathbb{P}^{2}
$$

with respect to the subbundle $\mathcal{O}_{C}\left(-2 w_{\infty}\right) \hookrightarrow \mathcal{O}_{C} \oplus \mathcal{O}_{C}$ (see Remark 3.4).

In order to give explicit coordinates for App, we fix a basis of rational functions

$$
\left\{\frac{1}{(x-t)}, \frac{x}{(x-t)}, \frac{y}{(x-t)}\right\}
$$

for the vector space $\mathrm{H}^{0}\left(C, \mathcal{O}_{C}\left(w_{\infty}+t_{1}+t_{2}\right)\right)$ and denote by $\boldsymbol{a}=\left(a_{0}: a_{1}: a_{2}\right)$ projective coordinates with respect to that basis.

Let $\boldsymbol{c}=\left(c_{0}: c_{1}: c_{2}\right) \in \mathbb{P}^{2}$ be coordinates of $\operatorname{Bun}^{-1}(\boldsymbol{z})$ with respect to the basis $\left\{\nabla^{0}(\boldsymbol{z}), \Theta_{1}^{0}(\boldsymbol{z}), \Theta_{2}^{0}(\boldsymbol{z})\right\}$ (see Section 3.4), that is, each $\nabla(\boldsymbol{z}) \in$ Bun $^{-1}(\boldsymbol{z})$ writes

$$
\nabla(\boldsymbol{z})=c_{0} \cdot \nabla^{0}(\boldsymbol{z})+c_{1} \cdot \Theta_{1}^{0}(\boldsymbol{z})+c_{2} \cdot \Theta_{2}^{0}(\boldsymbol{z}) .
$$

Since the subbundle $\mathcal{O}_{C}\left(-2 w_{\infty}\right) \hookrightarrow \mathcal{O}_{C} \oplus \mathcal{O}_{C}$ corresponds to the explicit section

$$
s: C \longrightarrow \mathcal{O}_{C} \oplus \mathcal{O}_{C} ;(x, y) \mapsto\left(\begin{array}{l}
1 \\
x
\end{array}\right)
$$

as in Remark 3.4, we can compute the coordinates of the mapping $A p p_{\boldsymbol{z}}: \mathbb{P}_{\boldsymbol{c}}^{2} \longrightarrow \mathbb{P}_{\boldsymbol{a}}^{2}$ :

$$
\operatorname{App}_{\boldsymbol{z}}(\boldsymbol{c})=a_{0} \cdot \frac{1}{(x-t)}+a_{1} \cdot \frac{x}{(x-t)}+a_{2} \cdot \frac{y}{(x-t)}
$$

where

$$
\begin{array}{lcccc}
a_{0}(\boldsymbol{c}) & = & r\left(\nu_{1}\left(2 z_{1}-t\right)+\nu_{2}\left(2 z_{2}-t\right)-t\right) \cdot c_{0} & -4 r z_{1}\left(z_{1}-t\right) \cdot c_{1} & -4 r z_{2}\left(z_{2}-t\right) \cdot c_{2} \\
a_{1}(\boldsymbol{c}) & = & -r\left(\nu_{1}+\nu_{2}-1\right) \cdot c_{0} & +4 r\left(z_{1}-t\right) \cdot c_{1} & +4 r\left(z_{2}-t\right) \cdot c_{2} \\
a_{2}(\boldsymbol{c}) & = & 2\left(\nu_{1}\left(z_{1}-t\right)-\nu_{2}\left(z_{2}-t\right)\right) \cdot c_{0} & -4\left(z_{1}-t\right)^{2} \cdot c_{1} & +4\left(z_{2}-t\right)^{2} \cdot c_{2}
\end{array}
$$

In other words, $A p p_{\boldsymbol{z}}: \mathbb{P}_{\boldsymbol{c}}^{2} \longrightarrow \mathbb{P}_{\boldsymbol{a}}^{2}$ is a projective transformation defined by a matrix with determinant

$$
\operatorname{det}\left(A p p_{\boldsymbol{z}}\right)=-32 r^{2}\left(t-z_{1}\right)^{2}\left(t-z_{2}\right)^{2}\left(\nu_{1}+\nu_{2}+1\right) .
$$

We promptly deduce that the rank drop exactly when $z_{1}=t$ or $z_{2}=t$. In addition, if $z_{1}=t$ and $z_{2} \neq t$, then $A p p_{\boldsymbol{z}}$ does not depend of $c_{1}$ and its matrix has rank two. The same happens if $z_{1} \neq t$ and $z_{2}=t$. If $z_{1}=z_{2}=t$, then the matrix has rank one. If $\nu_{1}+\nu_{2}-1 \neq 0$, a straightforward computation shows that the image point is $(t:-1: 0)$, which corresponds to a non-vanishing constant function in $\mathrm{H}^{0}\left(C, \mathcal{O}_{C}\left(w_{\infty}+t_{1}+t_{2}\right)\right)$. This shows that the image point is $w_{\infty}+t_{1}+t_{2} \in$ $\left|\mathcal{O}_{C}\left(w_{\infty}+t_{1}+t_{2}\right)\right|$ via the identification $\left|\mathcal{O}_{C}\left(w_{\infty}+t_{1}+t_{2}\right)\right| \simeq \mathbb{P H}^{0}\left(C, \mathcal{O}_{C}\left(w_{\infty}+\right.\right.$ $\left.\left.t_{1}+t_{2}\right)\right)$. Assertion (4) can easily be verified.

We now consider the case where

$$
\boldsymbol{Z}=\left(Z_{1}, Z_{2}\right)=\left(1 / z_{1}, 1 / z_{2}\right) \in U_{\infty} \subset X_{<} .
$$

Using the basis $\left\{\nabla^{\infty}, \Theta_{1}^{\infty}, \Theta_{2}^{\infty}\right\}$ instead of $\left\{\nabla^{0}, \Theta_{1}^{0}, \Theta_{2}^{0}\right\}$ one can find the coordinates of $A p p_{\boldsymbol{Z}}: \mathbb{P}_{\boldsymbol{C}}^{2} \longrightarrow \mathbb{P}_{\boldsymbol{a}}^{2}$ in terms of this basis:

$$
\operatorname{App}_{\boldsymbol{Z}}\left(C_{0} \cdot \nabla^{\infty}+C_{1} \cdot \Theta_{1}^{\infty}+C_{2} \cdot \Theta_{2}^{\infty}\right)=a_{0} \cdot \frac{1}{(x-t)}+a_{1} \cdot \frac{x}{(x-t)}+a_{2} \cdot \frac{y}{(x-t)}
$$

where

$$
\begin{aligned}
& a_{0}(\boldsymbol{C})=\quad \operatorname{tr}\left(1-\nu_{1}-\nu_{2}\right) \cdot C_{0} \quad+4 r\left(1-t Z_{1}\right) \cdot C_{1} \quad+4 r\left(1-t Z_{2}\right) \cdot C_{2} \\
& a_{1}(\boldsymbol{C})=r\left(\nu_{1}\left(2 t Z_{1}-1\right)+\nu_{2}\left(2 t Z_{2}-1\right)-1\right) \cdot C_{0}+4 r Z_{1}\left(t Z_{1}-1\right) \cdot C_{1}+4 r Z_{2}\left(t Z_{2}-1\right) \cdot C_{2} \\
& a_{2}(\boldsymbol{C})=2 t\left(\nu_{1}\left(t Z_{1}-1\right)-\nu_{2}\left(t Z_{2}-1\right)\right) \cdot C_{0}+4\left(t Z_{1}-1\right)^{2} \cdot C_{1}-4\left(t Z_{2}-1\right)^{2} \cdot C_{2}
\end{aligned}
$$


It follows that

$$
\operatorname{det}\left(A p p_{\boldsymbol{Z}}\right)=-32\left(t Z_{1}-1\right)^{2}\left(t Z_{2}-1\right)^{2}\left(\nu_{1}+\nu_{2}+1\right) .
$$

Since $U_{0} \cup U_{\infty}=\left(\mathbb{P}^{1} \times \mathbb{P}^{1}\right) \backslash\{(\infty, 0),(0, \infty)\}$, to conclude the proof of theorem, it remains to consider the case where $u=(\infty, 0)$ and $u=(0, \infty)$; we do not detail.

Theorem 3.19. If $\nu_{1}+\nu_{2}+1=0$, then the rational map

$$
\text { Bun } \times A p p: \overline{\operatorname{Con}_{1}^{\nu}(C, D)} \longrightarrow \mathbb{P}_{z_{1}}^{1} \times \mathbb{P}_{z_{2}}^{1} \times \mathbb{P}^{2}
$$

is non-dominant. Moreover, $(\text { Bun } \times A p p)^{-1}(y)$ is one dimensional for general $y$ lying on the image of Bun $\times$ App.

Proof. Assume $\nu_{1}+\nu_{2}+1=0$. If $z_{1} \neq t$ and $z_{2} \neq t$ then

$$
\operatorname{App}_{\boldsymbol{z}}\left(\nabla^{0}(\boldsymbol{z})\right)=-\frac{\nu_{1}}{2\left(z_{1}-t\right)} \operatorname{App}_{\boldsymbol{z}}\left(\Theta_{1}^{0}(\boldsymbol{z})\right)+\frac{\nu_{1}+1}{2\left(z_{2}-t\right)} \operatorname{App}_{\boldsymbol{z}}\left(\Theta_{2}^{0}(\boldsymbol{z})\right) .
$$

In addition, $A_{p p}\left(\Theta_{1}^{0}(\boldsymbol{z})\right)$ and $A p p_{\boldsymbol{z}}\left(\Theta_{2}^{0}(\boldsymbol{z})\right)$ do not coincide in $\mathbb{P}^{2}$. This shows that the image of $A p p_{\boldsymbol{z}}: \mathbb{P}^{2} \longrightarrow \mathbb{P}^{2}$ is the line spanned by $\operatorname{App}_{\boldsymbol{z}}\left(\Theta_{1}^{0}(\boldsymbol{z})\right)$ and $\operatorname{App}_{\boldsymbol{z}}\left(\Theta_{2}^{0}(\boldsymbol{z})\right)$.

3.8. Symplectic structure and Torelli phenomenon. Recall that the moduli space of connections $\operatorname{Con}^{\nu}(C, D)$ admits a canonical holomorphic symplectic structure, i.e. a holomorphic non degenerate 2-form $\omega: \omega \wedge \omega \neq 0$ at any point of $\operatorname{Con}^{\nu}(C, D)$ (see for instance [19]). Given any Lagrangian rational (or local) section $\nabla_{0}: \operatorname{Bun}^{\nu}(C, D) \rightarrow \operatorname{Con}^{\nu}(C, D)$, then the reduction map
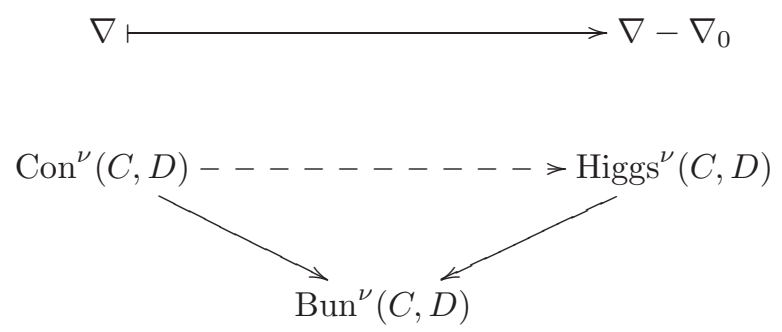

is symplectic. Moreover, the following three maps are Lagrangian

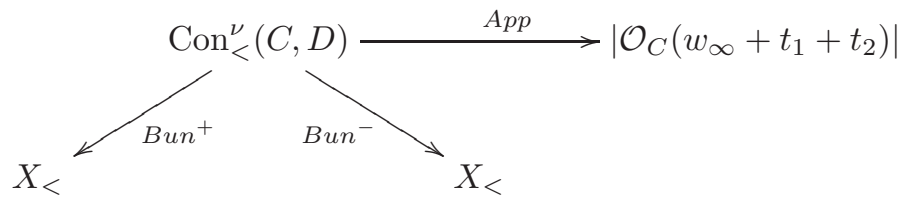

i.e. with Lagrangian fibers (see [19]). In particular, the section $\nabla^{0}$ defined in Section 3.4 is Lagrangian since it is a fiber of $\mathrm{Bun}^{-}$(see Remark 3.9). We promptly deduce from the reduction map $\nabla_{\boldsymbol{c}} \mapsto \nabla_{\boldsymbol{c}}-\nabla^{0}$ applied to the universal connection $\nabla_{\boldsymbol{c}}$ defined by (3.11) that the symplectic structure on $\operatorname{Con}^{\nu}(C, D)$ is given by $\omega=$ $d c_{1} \wedge d z_{1}+d c_{2} \wedge d z_{2}$ as for Higgs bundles in Theorem 3.6

The image of $\omega$ by the map Par: $\operatorname{Con}_{<}^{\nu}(C, D) \rightarrow S$ defined in (3.12) is given by setting $c_{i}=\nu_{i} / 2\left(z_{i}-\zeta_{i}\right), i=1,2$, i.e. by

$$
\omega=-\frac{1}{2}\left\{\nu_{1} \frac{d z_{1} \wedge d \zeta_{1}}{\left(z_{1}-\zeta_{1}\right)^{2}}+\nu_{2} \frac{d z_{2} \wedge d \zeta_{2}}{\left(z_{2}-\zeta_{2}\right)^{2}}\right\} .
$$


In particular, we see that, taking into account the symplectic structure of the bundle Bun: $\operatorname{Con}_{<}^{\nu}(C, D) \rightarrow \operatorname{Bun}_{<}^{\nu}(C, D)$, we can recover the eigenvalues $\nu_{1}, \nu_{2}$ in the spirit of Torelli Theorem.

Proposition 3.20. If we have an equivariant bundle symplectic isomorphism

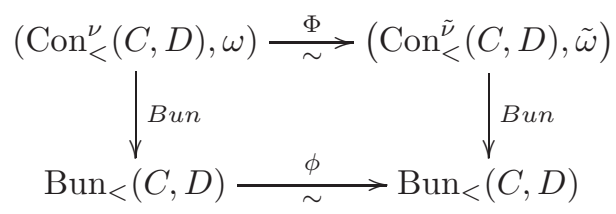

Then $\left(\tilde{\nu}_{1}, \tilde{\nu}_{2}\right)=\left(\nu_{1}, \nu_{2}\right)$ or $\left(\nu_{2}, \nu_{1}\right)$.

Proof. Any automorphism $\phi$ of $\operatorname{Bun}_{<}(C, D)=X_{<}=\mathbb{P}_{z_{1}}^{1} \times \mathbb{P}_{z_{2}}^{1}$ writes

$$
\left(z_{1}, z_{2}\right) \mapsto\left(\varphi\left(z_{1}\right), \psi\left(z_{2}\right)\right) \quad \text { or } \quad\left(\varphi\left(z_{2}\right), \psi\left(z_{1}\right)\right)
$$

for some Moebius transformations $\varphi, \psi$. The map $\phi_{D}:\left(z_{1}, z_{2}\right) \mapsto\left(z_{2}, z_{1}\right)$ admits a lifting

satisfying

$$
\Phi_{D}:\left(\left(z_{1}, \zeta_{1}\right),\left(z_{2}, \zeta_{2}\right)\right) \mapsto\left(\left(z_{2}, \zeta_{2}\right),\left(z_{1}, \zeta_{1}\right)\right)
$$

$$
\Phi_{D}^{*} \omega=-\frac{1}{2}\left\{\nu_{2} \frac{d z_{1} \wedge d \zeta_{1}}{\left(z_{1}-\zeta_{1}\right)^{2}}+\nu_{1} \frac{d z_{2} \wedge d \zeta_{2}}{\left(z_{2}-\zeta_{2}\right)^{2}}\right\}
$$

(it permutes $\nu_{1}$ and $\nu_{2}$ ). Now, considering $\phi$ isotopic to the identity, we will prove that its lifting $\Phi$ preserves coefficients $\nu_{1}$ and $\nu_{2}$. First, observe that $\phi$ admits a lifting preserving $\nu_{1}$ and $\nu_{2}$, namely

$$
\Phi:\left(\left(z_{1}, \zeta_{1}\right),\left(z_{2}, \zeta_{2}\right)\right) \mapsto\left(\left(\varphi\left(z_{1}\right), \varphi\left(\zeta_{1}\right)\right),\left(\psi\left(z_{2}\right), \psi\left(\zeta_{2}\right)\right)\right) .
$$

Indeed, we can decompose $\varphi$ and $\psi$ as a combination of $z \mapsto \alpha z, z \mapsto z+1$ and $z \mapsto 1 / z$, and easily check that, for each of these transformations, $\frac{d z \wedge d \zeta}{(z-\zeta)^{2}}$ is invariant, and therefore $\omega$ as well. We end up the proof by showing that there are no other equivariant bundle isomorphisms, i.e. if $\phi$ is the identity, then so is $\Phi$. Indeed, when we fix $z_{2}$, the $\Phi$ has to preserve the polar locus of $\omega$ (being symplectic), namely $\zeta_{2}=z_{2}$ and $\zeta_{1}=z_{1}$. In restriction to $\zeta_{2}=z_{2}$ (recall we have fixed $z_{2}$ ) $\Phi$ induces an automorphism of $\mathbb{P}_{z_{1}}^{1} \times \mathbb{P}_{\zeta_{1}}^{1}$ preserving the diagonal $\zeta_{1}=z_{1}$ and fixing $z_{1}$. It is therefore the identity. We easily conclude that $\Phi$ is the identity everywhere.

It is interesting to make the link with the approach of [13]. There, a birational model of the moduli of parabolic bundles was introduced by using apparent map in restriction to Higgs fields (see [13, Corollary 4.5]). Precisely, the restriction of apparent map to Higgs fields is given by

$$
\operatorname{App}_{\boldsymbol{z}}\left(c_{1} \cdot \Theta_{1}^{0}(\boldsymbol{z})+c_{2} \cdot \Theta_{2}^{0}(\boldsymbol{z})\right)=a_{0} \cdot \frac{1}{(x-t)}+a_{1} \cdot \frac{x}{(x-t)}+a_{2} \cdot \frac{y}{(x-t)}
$$

where

$$
\begin{array}{lcc}
a_{0}= & -r\left(c_{1} z_{1}\left(t-z_{1}\right)+c_{2} z_{2}\left(t-z_{2}\right)\right) \\
a_{1}= & r\left(c_{1}\left(t-z_{1}\right)+c_{2}\left(t-z_{2}\right)\right) \\
a_{2}= & c_{1}\left(t-z_{1}\right)^{2}+c_{2}\left(t-z_{2}\right)^{2}
\end{array}
$$

Therefore, the image $A_{p p}\left(\operatorname{Higgs}_{<}^{\nu}(C, D)\right) \subset\left|\mathcal{O}_{C}\left(w_{\infty}+t_{1}+t_{2}\right)\right|$ is given by $a_{0} b_{0}+$ $a_{1} b_{1}+a_{2} b_{2}=0$ where

$$
\begin{array}{ccc}
b_{0} & = & 2 t-z_{1}-z_{2} \\
b_{1} & = & t\left(z_{1}+z_{2}\right)-2 z_{1} z_{2} \\
b_{2} & = & r\left(z_{1}-z_{2}\right)
\end{array}
$$


and we get a natural birational map

$$
\text { Bun }^{\prime}: \operatorname{Bun}_{<}(C, D) \rightarrow\left|\mathcal{O}_{C}\left(w_{\infty}+t_{1}+t_{2}\right)\right|^{*} \simeq \mathbb{P}_{\boldsymbol{b}}^{2} ;\left(z_{1}, z_{2}\right) \mapsto\left(b_{0}: b_{1}: b_{2}\right) .
$$

This map blow-up the point $\left(z_{1}, z_{2}\right)=(t, t)$ and contracts the strict transform of the two lines $z_{1}=t$ and $z_{2}=t$. Another birational model of $\operatorname{Con}_{<}^{\nu}(C, D)$, more in the spirit of [13], is given by

$$
A p p \times \text { Bun }^{\prime}: \operatorname{Con}_{<}^{\nu}(C, D) \stackrel{\sim}{\sim} \mathbb{P}_{\boldsymbol{a}}^{2} \times \mathbb{P}_{\boldsymbol{b}}^{2}
$$

and we get (compare with [13, Theorem 1.1])

Proposition 3.21. If $\nu_{1}+\nu_{2}+1 \neq 0$, then the map 3.17) is birational and the image of the symplectic form is given by

$$
\omega=d \eta \quad \text { where } \eta=\left(\frac{\nu_{1}+\nu_{2}+1}{4}\right) \frac{a_{0} d b_{0}+a_{1} d b_{1}+a_{2} d b_{2}}{a_{0} b_{0}+a_{1} b_{1}+a_{2} b_{2}} .
$$

The polar locus of $\omega$ is supported by the incidence variety $a_{0} b_{0}+a_{1} b_{1}+a_{2} b_{2}=0$.

\section{Appendix}

4.1. Parabolic vector bundles. Let $C$ be a smooth irreducible projective complex curve and $D=t_{1}+\cdots+t_{n}$ be a reduced divisor supported on $n$ distinct points $\left\{t_{1}, \ldots, t_{n}\right\} \subset C$. A rank two quasi-parabolic vector bundle on $(C, D)$ is the data $(E, \mathbf{p})$ where $E$ is a rank two holomorphic vector bundle over $C$ and $\mathbf{p}=\left\{p_{1}, \ldots, p_{n}\right\}$ are given 1-dimensional subspaces $p_{k} \subset E_{t_{k}}$ for $k \in\{1, \ldots, n\}$. An isomorphism between quasi-parabolic vector bundles is, by definition, an isomorphism between underlying vector bundles preserving parabolic directions. A parabolic vector bundle is a quasi-parabolic vector bundle together with a collection of weights $\mu=\left(\mu_{1}, \ldots, \mu_{n}\right) \in[0,1]^{n}$. It allows us to introduce a notion of stability in order to introduce a good moduli space. Given a line bundle $L \subset E$, the $\mu$-stability index of $L$ is the real number

$$
\operatorname{Stab}(L):=\operatorname{deg} E-2 \operatorname{deg} L+\sum_{p_{k} \neq L_{t_{k}}} \mu_{k}-\sum_{p_{k}=L_{t_{k}}} \mu_{k} .
$$

Definition 4.1. A parabolic vector bundle $(E, \mathbf{p})$ is called $\mu$-stable (resp. $\mu$ semistable) if for any rank one subbundle $L \subset E$, the following inequality holds

$$
\operatorname{Stab}(L)>0 \quad(\text { resp. } \operatorname{Stab}(L) \geq 0) .
$$

It is well known, see [16], that the moduli space of $\mu$-semistable parabolic vector bundles with fixed determinant line bundle is a normal irreducible projective variety. The open subset of $\mu$-stable parabolic bundles is smooth. We note that the stability index of $L \subset E$ is zero if, and only if, the weights lie along the following hyperplane in $[0,1]^{n}$

$$
\operatorname{deg}(E)-2 \operatorname{deg} L+\sum_{p_{k} \neq L_{t_{k}}} \mu_{k}-\sum_{p_{k}=L_{t_{k}}} \mu_{k}=0 .
$$

Each one of these hyperplanes is called a wall. If we cut out $[0,1]^{n}$ by all possible walls, one gets in the complement of finitely many irreducible connected components, which are called chambers. In each chamber, any $\mu$-semistable parabolic vector bundle is $\mu$-stable, and the moduli space is constant, that is, it is independent of $\mu$. Nevertheless, it can be empty. When we have two adjacent chambers separated by a wall, then there is a locus of $\mu$-stable parabolic bundles in each 
chamber that became unstable when we cross the wall. Along the wall, we identify strictly semistable parabolic bundles $(E, \mathbf{p})$ and $\left(E^{\prime}, \mathbf{p}^{\prime}\right)$ with $\operatorname{gr}(E, \mathbf{p})=\operatorname{gr}\left(E^{\prime}, \mathbf{p}^{\prime}\right)$, see [16, Section 4]. Over the projective line, the description of the moduli space of quasi-parabolic bundles has been done in [3].

4.2. Elementary transformations. In the construction of the moduli space of quasi-parabolic bundles, the determinant line bundle is fixed. Actually, up to twists and elementary transformations, we can choose the determinant bundle arbitrarily, for instance the trivial line bundle $\mathcal{O}_{C}$. Twists preserve the parity of the determinant and elementary transformations change it. We start by recalling what is an elementary transformation as well as its main properties. Given $t \in C$ and a direction $p \subset E_{t}$ the vector bundle $E^{-}$is defined by the following exact sequence of sheaves

$$
0 \longrightarrow E^{-} \longrightarrow E \longrightarrow E / p \longrightarrow 0
$$

where $p$ appearing above is considered as a sky-scrapper sheaf. The new parabolic direction $p^{-} \subset E^{-}$is the kernel of the morphism $E^{-} \longrightarrow E$. By identifying sections of $E$ and $E^{-}$outside of $t$, one obtains a birational bundle transformation

$$
\text { elem }_{t}^{-}: E \rightarrow E^{-}
$$

with center at $p$, which is an isomorphism outside $t$. We shall say that it is a negative elementary transformation. At a neighborhood of $t$, it can be described as follows. We can choose a local trivialization $\left.E\right|_{U} \simeq U \times \mathbb{C}^{2}$ such that

$$
p=\left(\begin{array}{l}
0 \\
1
\end{array}\right) \subset E_{t} .
$$

and elem ${ }_{t}^{-}:\left.\left.E\right|_{U} \rightarrow E^{-}\right|_{U}$ is given by

$$
\text { elem }_{t}^{-}(x, Y)=\left(\begin{array}{cc}
1 / x & 0 \\
0 & 1
\end{array}\right) \cdot Y \text {. }
$$

From the point of view of ruled surfaces, it corresponds to a flip with center at $[p] \in \mathbb{P} E$, that is, a blow up at $[p]$ followed by a contraction of the old fiber. If the direction $p$ is contained in a line bundle $L \subset E$, then it is left unchanged and one obtains a line bundle $L \subset E^{-}$. If $L \subset E$ does not contain $p$, then we get $L^{-} \subset E$ where

$$
L^{-}=L \otimes \mathcal{O}_{C}(-t) .
$$

In addition, we have the following property

$$
\operatorname{det}\left(E^{-}\right)=\operatorname{det}(E) \otimes \mathcal{O}_{C}(-t) .
$$

When we perform an elementary transformation, the stability condition is preserved after an appropriate modification of weights. If $(E, \mathbf{p})$ is $\mu$-stable and we perform an elementary transformation elem $_{t_{k}}^{-}$, then $\left(E^{-}, \mathbf{p}^{-}\right)$is $\mu^{\prime}$-stable where

$$
\left\{\begin{array}{l}
\mu_{k}^{\prime}=1-\mu_{k} \\
\mu_{j}^{\prime}=\mu_{j} \quad j \neq k
\end{array}\right.
$$

If $\operatorname{Bun}_{L}^{\mu}(C, D)$ denotes the moduli space of $\mu$-semistable parabolic vector bundles with fixed determinant line bundle $L$, then $e l e m_{t_{k}}^{-}$defines an isomorphism between moduli spaces

$$
\begin{aligned}
\operatorname{elem}_{t_{k}}^{-}: \operatorname{Bun}_{L}^{\mu}(C, D) & \longrightarrow \operatorname{Bun}_{L\left(-t_{k}\right)}^{\mu^{\prime}}(C, D) \\
(E, \mathbf{p}) & \mapsto \operatorname{elem}_{t_{k}}^{-}(E, \mathbf{p}) .
\end{aligned}
$$


We can define a positive elementary transformation elem $_{t}^{+}$as

$$
\text { elem }_{t}^{+}:=\mathcal{O}_{C}(t) \otimes \text { elem }_{t}^{-}: E \rightarrow E^{+}
$$

where $E^{+}=E^{-} \otimes \mathcal{O}_{C}(t)$. It is the inverse of $e l e m_{t}^{-}$. As before, stability condition is preserved by elementary positive transformations, with the same modification of weights.

4.3. Endomorphisms of quasi-parabolic vector bundles. The space of global endomorphisms of a rank two vector bundle as well as the automorphism group are well known, see for example [15, Theorem 1]. In this section, we study the space of traceless endomorphisms of a quasi-parabolic bundles over an elliptic curve.

Let $(E, \mathbf{p})$ be a quasi-parabolic bundle. We say that an endomorphism $\sigma \in$ $\operatorname{End}(E)$ fixes the parabolic structure $\mathbf{p}$ if $\sigma\left(p_{k}\right) \subset p_{k}$, for all $k=1, \ldots, n$. Let $\operatorname{End}(E, \mathbf{p})$ be the vector space of endomorphisms fixing the parabolic structure $\mathbf{p}$ and $\operatorname{End}_{0}(E, \mathbf{p})$ its subspace of traceless endomorphisms. We notice that we have a canonical decomposition:

$$
\operatorname{End}(E, \mathbf{p})=<I d>\oplus \operatorname{End}_{0}(E, \mathbf{p})
$$

where $I d \in \operatorname{End}(E, \mathbf{p})$ is the identity. This follows from the following simple remark: if $A$ is a $2 \times 2$ matrix then

$$
A=\frac{\operatorname{tr}(A)}{2} \cdot I d+\left(A-\frac{\operatorname{tr}(A)}{2} \cdot I d\right) .
$$

Lemma 4.2. Let $E=\mathcal{O}_{C} \oplus L$ be a rank 2 bundle over a projective smooth curve $C$ with $\mathcal{L}$ of nonnegative degree. The following statements hold true.

(a) If $L \supsetneqq \mathcal{O}_{C}$ then

$$
\operatorname{End}_{0}(E)=\left\{\left(\begin{array}{cc}
a & 0 \\
\gamma & -a
\end{array}\right) ; a \in \mathbb{C}, \gamma=\left\{\gamma_{i}\right\} \in \mathrm{H}^{0}(C, L)\right\}
$$

(b) If $\mathcal{L}=\mathcal{O}_{C}$ then

$$
\operatorname{End}_{0}(E)=\left\{\left(\begin{array}{cc}
a & b \\
c & -a
\end{array}\right) ; a, b, c \in \mathbb{C}\right\}
$$

Proof. We leave the proof to the reader; see for example [15, Theorem 1].

We recall that a quasi-parabolic bundle $(E, \mathbf{p})$ is decomposable if there exists a decomposition $E=L_{1} \oplus L_{2}$ such that each parabolic direction is contained in $L_{1}$ or $L_{2}$. In this case, we write

$$
(E, \mathbf{p})=\left(L_{1}, \mathbf{p}_{\mathbf{1}}\right) \oplus\left(L_{2}, \mathbf{p}_{\mathbf{2}}\right) .
$$

4.4. The case of elliptic curves. In what follows we will determine the traceless endomorphisms of an indecomposable quasi-parabolic bundle over an elliptic curve. This will be useful to assure existence of logarithmic connections. Before that, we shall give one example.

Example 4.1. Let $\left(\mathcal{O}_{C} \oplus \mathcal{O}_{C}\left(t_{1}\right),\left\{p_{1}, p_{2}\right\}\right)$ be a quasi-parabolic bundle over an elliptic curve $\left(C,\left\{t_{1}, t_{2}\right\}\right)$ with

(1) $p_{1}$ outside $\left.\mathcal{O}_{C}\right|_{t_{1}}$ and $\left.\mathcal{O}_{C}\left(t_{1}\right)\right|_{t_{1}}$; and

(2) $\left.p_{2} \subset \mathcal{O}_{C}\left(t_{1}\right)\right|_{t_{2}}$. 
It is indecomposable because any subbundle given by an embedding of the trivial bundle $\mathcal{O}_{C} \hookrightarrow \mathcal{O}_{C} \oplus \mathcal{O}_{C}\left(t_{1}\right)$ corresponds to a section of $\mathbb{P}\left(\mathcal{O}_{C} \oplus \mathcal{O}_{C}\left(t_{1}\right)\right)$ which has $(1: 0)$ as a base point over $t_{1}$. In fact, since $C$ is elliptic $h^{0}\left(\mathcal{O}_{C}\left(t_{1}\right)\right)=1$. On the other hand, if $\gamma \in \mathrm{H}^{0}\left(C, \mathcal{O}_{C}\left(t_{1}\right)\right)$ is a section which corresponds to the divisor $D=t_{1}$ then

$$
\operatorname{End}_{0}(E, \mathbf{p})=\left\{\left(\begin{array}{cc}
0 & 0 \\
c \gamma & 0
\end{array}\right) ; c \in \mathbb{C}\right\} .
$$

Proposition 4.3. Let $E=\mathcal{O}_{C} \oplus L, \operatorname{deg}(L) \geq 0$, be a decomposable rank 2 bundle over an elliptic curve $C$. Assume $(E, \boldsymbol{p})$ is indecomposable but not simple, i.e. $\operatorname{End}_{0}(E, \boldsymbol{p}) \neq\{0\}$. Then we are in the following case

(1) $\operatorname{End}_{0}(E, \boldsymbol{p})=\mathbb{C}$

(2) the support $D$ of parabolics splits as $D=D_{0}+D_{1}$ with $\operatorname{deg}\left(D_{1}\right)>0$,

(3) $L \simeq \mathcal{O}_{C}\left(D_{1}\right)$ (and has $>0$ degree),

(4) parabolics over $D_{0}$ are lying on $L$,

(5) parabolics over $D_{1}$ are outside $L$ and generic.

Here, generic means that there is no embedding $\mathcal{O}_{C} \hookrightarrow E$ passing through all parabolics over $D_{1}$, i.e. $(E, \boldsymbol{p})$ is indecomposable.

Observe that, by Lemma 4.2 one can find an embedding $\mathcal{O}_{C} \hookrightarrow E$ passing through all but one parabolics over $D_{1}$. In particular, given the decomposition $D=D_{0}+D_{1}$, there is a unique such parabolic bundle $(E, \mathbf{p})$ up to isomorphism.

Proof. If $L=\mathcal{O}_{C}$ and $(E, \mathbf{p})$ is indecomposable, then there are at least 3 parabolics which not two of them lies in the same embedding of $\mathcal{O}_{C} \hookrightarrow \mathcal{O}_{C} \oplus \mathcal{O}_{C}$. Then it follows from Lemma 4.2 item $(b)$ that

$$
\operatorname{End}_{0}\left(\mathcal{O}_{C} \oplus \mathcal{O}_{C}, \mathbf{p}\right)=\{0\} .
$$

Let us suppose $L \nsubseteq \mathcal{O}_{C}$ and let $\phi$ be a traceless endomorphism that fixes the parabolics. Lemma 4.2 item $(a)$ implies that we can choose a covering of $C$ and trivializations such that the vector $e_{1}$ generates $\mathcal{O}_{C}, e_{2}$ generates $L$ and

$$
\phi=\left(\begin{array}{cc}
a & 0 \\
\gamma & -a
\end{array}\right)
$$

where $a \in \mathbb{C}, \gamma \in \mathrm{H}^{0}(C, L)$. If $a \neq 0$, then we see that the locus of fixed points of $\phi$ outside $L$ defines a section of $E$

$$
\left(\begin{array}{c}
1 \\
\frac{\gamma}{2 a}
\end{array}\right)
$$

which generates a subbundle $\mathcal{O}_{C} \hookrightarrow E$ containing all parabolics outside $L$, showing that $(E, \mathbf{p})$ must be decomposable. We can thus assume $a=0$.

Since $\phi$ preserves the parabolic structure, we have for each parabolic $p_{k}$

- either $p_{k}$ is in the subbundle $L \subset E$,

- or the support $t_{k}$ of $p_{k}$ is a zero of $\gamma$.

Therefore, we can decompose $D=D_{0}+D_{1}$ where $D_{0}$ is the support of those $p_{k}$ 's lying in $L$.

Since $(E, \mathbf{p})$ is indecomposable, there is no embedding $\mathcal{O}_{C} \hookrightarrow E$ passing through all parabolics over $D_{1}$. Assume that our decomposition $E=\mathcal{O}_{C} \oplus L$ maximize the number of parabolics lying on the first factor. Set $D_{1}=D_{1}^{\prime}+D_{1}^{\prime \prime}$ with $D_{1}^{\prime}$ supporting those parabolics in $\mathcal{O}_{C}$ and $\operatorname{deg}\left(D_{1}^{\prime \prime}\right)>0$. By maximality, we have that 
each section $\varphi \in H^{0}(C, L)$ which vanishes on $D_{1}^{\prime}$ automatically vanishes on $D_{1}^{\prime \prime}$. In other words, each section of $L \otimes \mathcal{O}_{C}\left(-D_{1}^{\prime}\right)$ automatically vanishes on $D_{1}^{\prime \prime}$. On the other hand, we know that $L \otimes \mathcal{O}_{C}\left(-D_{1}^{\prime}\right)$ admits a non zero section defined by $\gamma$. But on the elliptic curve $C$, the only linear systems with base points are of the form $\left|t_{k}\right|$, meaning that $L \otimes \mathcal{O}_{C}\left(-D_{1}^{\prime}\right) \simeq \mathcal{O}_{C}\left(t_{k}\right)$ and $D_{1}^{\prime \prime}=t_{k}$ reduces to a single point. We therefore deduce that $L \simeq \mathcal{O}_{C}\left(D_{1}\right)$ and all parabolics but $p_{k}$, over $D_{1}$, are lying on the first factor $\mathcal{O}_{C} \subset E$.

Let $E_{0}$ be the unique indecomposable rank 2 bundle, over an elliptic curve, with trivial determinant and having $\mathcal{O}_{C}$ as maximal subbundle. It corresponds to the non trivial extension defined by the following exact sequence

$$
0 \longrightarrow \mathcal{O}_{C} \longrightarrow E_{0} \longrightarrow \mathcal{O}_{C} \longrightarrow 0 \text {. }
$$

Proposition 4.4. Let $\left(E_{0}, \boldsymbol{p}\right)$ be a quasi-parabolic bundle, where $E_{0}$ is the indecomposable bundle as above.

(1) If all parabolics lie in the maximal subbundle $\mathcal{O}_{C} \hookrightarrow E_{0}$ then

$$
\operatorname{End}_{0}\left(E_{0}, \boldsymbol{p}\right) \simeq \mathbb{C}
$$

(2) If there exists at least one parabolic outside $\mathcal{O}_{C} \hookrightarrow E_{0}$ then

$$
\operatorname{End}_{0}\left(E_{0}, \boldsymbol{p}\right)=\{0\} .
$$

Proof. The traceless endomorphism space of $E_{0}$ is given by

$$
\operatorname{End}_{0}\left(E_{0}\right)=\left\{\left(\begin{array}{ll}
0 & b \\
0 & 0
\end{array}\right) ; b \in \mathbb{C}\right\} .
$$

Here we are considering that in local charts $U_{i} \subset C, e_{1}$ generates the maximal subbundle $\mathcal{O}_{C}$. Then any traceless endomorphism leaves $\mathcal{O}_{C}$ invariant. Also if a parabolic direction outside $\mathcal{O}_{C}$ is fixed, one gets $b=0$. This is enough to conclude the proof of proposition.

Proposition 4.5. Let $(E, \boldsymbol{p})$ be indecomposable but not simple rank 2 quasiparabolic bundle over an elliptic curve $C$. Assume $E$ has trivial determinant line bundle. Then, up to elementary transformations and twists, we can assume $E=E_{0}$ with all the parabolic lying in the maximal subbundle $\mathcal{O}_{C} \hookrightarrow E_{0}$.

Proof. The proof follows essentially from Proposition 4.3 and Proposition 4.4. Suppose $E$ is decomposable, $E=M^{-1} \oplus M$, deg $M=k \geq 0$. Then $\mathbb{P} E=\mathbb{P}\left(\mathcal{O}_{C} \oplus M^{2}\right)$. From Proposition 4.3, the support $D$ of parabolics splits as $D=D_{0}+D_{1}$ with $\operatorname{deg}\left(D_{1}\right)>0$ and $\mathcal{O}_{C}\left(D_{1}\right)=M^{2}$. Parabolics over $D_{1}$ are outside $M \hookrightarrow E$ (which corresponds to $\left.M^{2} \hookrightarrow \mathcal{O}_{C} \oplus M^{2}\right)$ ) and generic. Parabolics over $D_{0}$ are lying on $M$. After a composition elem $D_{D_{1}}$ of $2 k$ elementary transformation over $D_{1}$ and twist (to get trivial determinant), we arrive in $E_{0}$ with all the parabolics lying in the maximal subbundle. If $E$ is indecomposable, the conclusion follows from Proposition 4.4.

4.5. Moduli space of connections. Let $C$ be a smooth projective curve and $D=t_{1}+\cdots+t_{n}$ be a reduced divisor on $C, n \geq 1$. We will fix the data in order to introduce the moduli space of connections. Firstly, let us fix a degree $d$ line bundle $L_{0}$ over $C$. We also set a local exponent $\nu \in \mathbb{C}^{2 n}$ satisfying the Fuchs relation

$$
\sum_{k=1}^{n}\left(\nu_{k}^{+}+\nu_{k}^{-}\right)+d=0
$$


and the generic condition $\nu_{1}^{\epsilon_{1}}+\cdots+\nu_{n}^{\epsilon_{n}} \notin \mathbb{Z}$ for any $\epsilon_{k} \in\{+,-\}$, to avoid reducible connections. Let $\zeta: L_{0} \longrightarrow L_{0} \otimes \Omega_{C}^{1}(D)$ be any fixed rank one logarithmic connection on $L_{0}$ satisfying

$$
\operatorname{Res}_{t_{k}}(\zeta)=\nu_{k}^{+}+\nu_{k}^{-}
$$

for all $k=1, \ldots, n$. We denote by $\operatorname{Con}^{\nu}(C, D)$ the moduli space of triples $(E, \nabla, \mathbf{p})$ where

(1) $(E, \mathbf{p})$ is a rank 2 quasi-parabolic vector bundle over $(C, D)$ having $L_{0}$ as determinant bundle;

(2) $\nabla: E \longrightarrow E \otimes \Omega_{C}^{1}(D)$ is a logarithmic connection on $E$ with polar divisor $D$, having $\nu$ as local exponents and $\operatorname{tr}(\nabla)=\zeta$;

(3) two triples $(E, \nabla, \mathbf{p})$ and $\left(E^{\prime}, \nabla^{\prime}, \mathbf{p}\right)$ are equivalent when there is an isomorphism between quasi-parabolic bundles $(E, \mathbf{p})$ and $\left(E^{\prime}, \mathbf{p}^{\prime}\right)$ conjugating $\nabla$ and $\nabla^{\prime}$.

Actually, in order to obtain a good moduli space we need a stability condition. A tripe $(E, \nabla, \mathbf{p})$ is called $\mu$-stable (resp. $\mu$-semistable) if for any $\nabla$-invariant line bundle $L \subset E$, we have

$$
\operatorname{Stab}(L)>0 \quad(\text { resp. } \operatorname{Stab}(L) \geq 0)
$$

(see Definition 4.1). But an invariant line bundle $L$ would force a relation

$$
\nu_{1}^{\epsilon_{1}}+\cdots+\nu_{n}^{\epsilon_{n}}+\operatorname{deg}(L)=0
$$

which is obtained by applying Fuchs relation to the restriction $\left.\nabla\right|_{L}$. This contradicts our hypothesis on $\nu$. Therefore under generic condition on the local exponent all the connections arising in our moduli space are stable. It follows from 17, Theorem 3.5] that $\operatorname{Con}^{\nu}(C, D)$ is a quasi-projective variety.

A priori, $\operatorname{Con}^{\nu}(C, D)$ depends on the choice of $L_{0}$. But up to twists, we can go into either the even case $L_{0}=\mathcal{O}_{C}$ or the odd case $L_{0}=\mathcal{O}_{C}(t)$. In fact, given a rank one logarithmic connection $\eta: L \longrightarrow L \otimes \Omega_{C}^{1}(D)$ with local exponents $\left(k_{1}, \ldots, k_{n}\right)$, we can define a twisting map

$$
\begin{aligned}
\otimes(L, \eta): \mathrm{Con}^{\nu}(C, D) & \longrightarrow \mathrm{Con}^{\nu^{\prime}}(C, D) \\
(E, \nabla, \mathbf{p}) & \mapsto(E \otimes L, \nabla \otimes \eta, \mathbf{p})
\end{aligned}
$$

where $\nu^{\prime}=\left(\nu_{1}^{ \pm}+k_{1}, \ldots, \nu_{n}^{ \pm}+k_{n}\right)$. Such map is an isomorphism between moduli spaces, in particular our moduli space only depend on differences $\nu_{k}^{+}-\nu_{k}^{-}$. In the even case, we can assume that $\left(L_{0}, \zeta\right)=\left(\mathcal{O}_{C}, d\right)$ where $d$ means the trivial rank one connection. In the odd case, one may suppose $\left(L_{0}, \zeta\right)=\left(\mathcal{O}_{C}(t), d-\frac{d x}{x-t}\right)$.

Now let us deal with elementary transformations. When we perform a transformation $e l e m_{t_{k}}^{-}:(E, \mathbf{p}) \rightarrow\left(E^{-}, \mathbf{p}^{-}\right)$the new connection $\nabla^{-}$on $E^{-}$has local exponents

$$
\left(\nu_{k}^{+}, \nu_{k}^{-}\right)^{\prime}=\left(\nu_{k}^{-}+1, \nu_{k}^{+}\right)
$$

and the other $\nu_{j}, j \neq k$, are left unchanged. Finally, we can go from the odd to the even case by performing one negative elementary transformation elem $_{t_{n}}^{-}$.

In the case we are interested in, $C$ is supposed to be an elliptic curve and $D=$ $t_{1}+t_{2}$. For computation, we can assume $C \subset \mathbb{P}^{2}$ is the smooth projective cubic curve

$$
z y^{2}=x(x-z)(x-\lambda z)
$$


with $\lambda \in \mathbb{C}, \lambda \neq 0,1$. And by the above digression one can set $L=\mathcal{O}_{C}\left(w_{\infty}\right)$, $w_{\infty}=(0: 1: 0)$. As local exponents, we can take

$$
\left(\nu_{1}^{ \pm}, \nu_{2}^{ \pm}\right)=\left( \pm \frac{\nu_{1}}{2}-\frac{1}{2}, \pm \frac{\nu_{2}}{2}\right) .
$$

We note that for each $k \in\{1,2\}$, the condition $\nu_{k}^{+}=\nu_{k}^{-}$is equivalent to $\nu_{k}=0$.

Acknowledgements. The first author would like thanks the Institut de Recherche en Mathématique de Rennes, IRMAR, for the hospitality and support.

\section{REFERENCES}

[1] D. Arinkin and S. Lysenko. On the moduli spaces of SL(2)-bundles with connections on $\mathbb{P}^{1} \backslash\left\{x_{1}, \ldots, x_{4}\right\}$. Int. Math. Research Notices (IRMN), 19 (1997) 983-999.

[2] M. F. Atiyah, Complex analytic connections in fibre bundles. Trans. Amer. Math. Soc. 85 (1957) 181-207.

[3] S. Bauer, Parabolic bundles, elliptic surfaces and SU(2)-representation spaces of genus zero Fuchsian groups. Math. Ann. 290 (1991) 509-526.

[4] I. Biswas, A criterion for the existence of a flat connection on a parabolic vector bundle. Advances in Geometry 2 (2002) 231-241.

[5] P. Boalch, Symplectic manifolds and isomonodromic deformations. Adv. Math. 163 (2001) 137-205.

[6] N. Fernández Vargas, Geometry of the moduli of parabolic bundles on elliptic curves. Trans. of the AMS (to appear). arXiv:1611.05417

[7] V. Heu and F. Loray, Flat rank 2 vector bundles on genus 2 curves. Memoirs of the AMS (to appear). arXiv:1401.2449

[8] M. Inaba, Moduli of parabolic connections on curves and the Riemann-Hilbert correspondence. J. Algebraic Geom. 22 (2013) 407-480.

[9] M. Inaba, K. Iwasaki and M.-H. Saito, Dynamics of the sixth Painlevé equation. Théories asymptotiques et équations de Painlevé, 103-167, Sémin. Congr., 14, Soc. Math. France, Paris, 2006.

[10] M. Inaba, K. Iwasaki and M.-H. Saito, Moduli of stable parabolic connections, RiemannHilbert correspondence and geometry of Painlevé equation of type VI. I. Publ. Res. Inst. Math. Sci. 42 (2006) 987-1089.

[11] A. Komyo and M.-H. Saito, Explicit description of jumping phenomena on moduli spaces of parabolic connections and Hilbert schemes of points on surfaces. Kyoto J. Math. (to appear). arXiv:1611.00971

[12] F. Loray, Isomonodromic deformations of Lamé connections, the Painlevé VI equation and Okamoto symmetry. Izv. Math. 80 (2016) 113-166.

[13] F. Loray and M.-H. Saito, Lagrangian fibrations in duality on moduli spaces of rank 2 logarithmic connections over the projective line. Int. Math. Res. Not. IMRN 2015 (2015) 995-1043.

[14] M. Maruyama, On Classification of ruled surfaces. Lect. in Math. Dept. Math. Kyoto Univ. 3, Kinokuniya, Tokyo (1970).

[15] M. Maruyama, On automorphism groups of ruled surfaces. J. Math. Kyoto Univ. 11 (1971) 89-112.

[16] V. B. Mehta and C. S. Seshadri, Moduli of vector bundles on curves with parabolic structures. Math. Ann. 248 (1980) 205-239.

[17] N. Nitsure, Moduli of semistable logarithmic connections. Journal Amer. Math. Sci. 6 (1993) 597-609.

[18] S. Oblezin, Isomonodromic deformations of $\mathfrak{s l}(2)$ Fuchsian systems on the Riemann sphere. Mosc. Math. J. 5 (2005) 415-441.

[19] C. Simpson, Iterated destabilizing modifications for vector bundles with connection. Vector bundles and complex geometry, 183-206, Contemp. Math., 522, Amer. Math. Soc., Providence, RI, 2010.

[20] S. Szabó, Deformation theory of Fuchsian equations and logarithmic connections. Studia Sci. Math. Hungar. 49 (2012) 466-481.

[21] A. Weil, Généralisation des fonctions abéliennes. J. Math. Pures Appl. 17 (1938) 47-87. 
Universidade Federal Fluminense, Rua Mário Santos Braga S/N, Niterói, RJ, Brasil

Email address: tfassarella@id.uff.br

Univ Rennes 1, CNRS, Institut de Recherche en Mathématique de Rennes, iRmaR, UMR 6625, Rennes, France

Email address: frank.loray@univ-rennes1.fr 